\title{
State-of-the-art loV trust management A meta-synthesis systematic literature review (SLR)
}

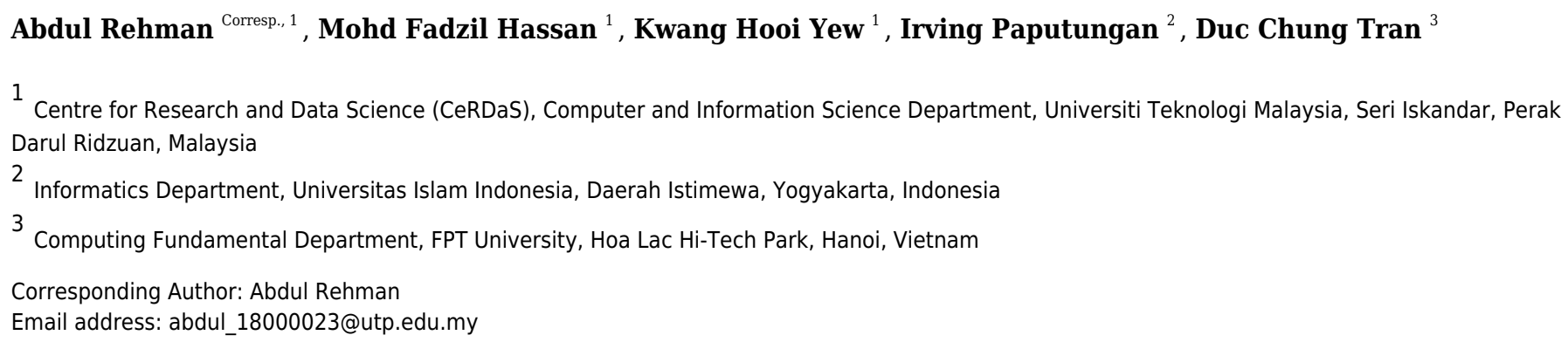

In the near future, the Internet of Vehicles (IoV) is foreseen to become an inviolable part of smart cities. The integration of vehicular ad hoc networks (VANETs) into the loV is being driven by the advent of the Internet of Things (IOT) and high-speed communication. However, both the technological and non-technical elements of loV need to be standardized prior to deployment on the road. This study focuses on trust management (TM) in the IoV/VANETs/ITS (intelligent transport system). Trust has always been important in vehicular networks to ensure safety. A variety of techniques for TM and evaluation have been proposed over the years, yet few comprehensive studies that lay the foundation for the development of a "standard" for TM in loV have been reported. The motivation behind this study is to examine all the trust models available for vehicular networks to bring together all the techniques from previous studies in this review. The study was carried out using a systematic method in which 31 papers out of 256 research publications were screened. An in-depth analysis of all the trust models was conducted and the strengths and weaknesses of each are highlighted. Considering that solutions based on Al are necessary to meet the requirements of a smart city, our second objective is to analyze the implications of incorporating an Al method based on "context awareness" in a vehicular network. It is evident from mobile ad hoc networks (MANETs) that there is potential for context awareness in ad hoc networks. The findings are expected to contribute significantly to the future formulation of IoVITS standards. In addition, gray areas and open questions for new research dimensions are highlighted. 


\section{State-of-the-Art loV Trust Management}

\section{A Meta-Synthesis Systematic Literature Review (SLR)}

3

4 Abdul Rehman ${ }^{1}$, Mohd Fadzil Hassan ${ }^{1}$, Kwang Hooi Yew ${ }^{1}$, Irving Paputungan ${ }^{2}$, Duc Chung

$5 \operatorname{Tran}^{3}$

6

$7 \quad{ }^{1}$ Centre for Research and Data Science (CeRDaS), Computer and Information Science

8 Department, Universiti Teknologi PETRONAS, 32610, Seri Iskandar, Perak Darul Ridzuan,

9 Malaysia.

102 Informatics Department, Universitas Islam Indonesia, Daerah Istimewa Yogyakarta 55584,

11 Indonesia

$12{ }^{3}$ Computing Fundamental Department, FPT University, Hoa Lac Hi-Tech Park, Hanoi, Vietnam, $13 \quad 155300$

Corresponding Author:

16 Abdul Rehman

17 Computer and Information Science Department, Universiti Teknologi PETRONAS, 32610, Seri

18 Iskandar, Perak Darul Ridzuan, Malaysia.

19 abdul 18000023@utp.edu.my 


\section{Abstract}

42 In the near future, the Internet of Vehicles (IoV) is foreseen to become an inviolable part of smart cities. The integration of vehicular ad hoc networks (VANETs) into the IoV is being driven by the advent of the Internet of Things (IoT) and high-speed communication. However, both the technological and non-technical elements of IoV need to be standardized prior to deployment on the road. This study focuses on trust management (TM) in the IoV/VANETs/ITS (intelligent transport system). Trust has always been important in vehicular networks to ensure safety. A variety of techniques for TM and evaluation have been proposed over the years, yet few comprehensive studies that lay the foundation for the development of a "standard" for TM in IoV have been reported. The motivation behind this study is to examine all the trust models available for vehicular networks to bring together all the techniques from previous studies in this review. The study was carried out using a systematic method in which 31 papers out of 256 research publications were screened. An in-depth analysis of all the trust models was conducted and the strengths and weaknesses of each are highlighted. Considering that solutions based on AI are necessary to meet the requirements of a smart city, our second objective is to analyze the implications of incorporating an artificial intelligence (AI) method based on "context awareness" in a vehicular network. It is evident from mobile ad hoc networks (MANETs) that there is potential for context awareness in ad hoc networks. The findings are expected to contribute significantly to the future formulation of IoVITS standards. In addition, gray areas and open questions for new research dimensions are highlighted.

\section{Introduction}

After the rise of the Internet of Things (IoT), several new technologies have merged to form vehicular ad hoc networks (VANETs), which have evolved significantly over the last decade. The IoT has attracted researchers' attention in that it enables the unification of VANETs with the Internet (Alam et al. 2015; Hamid et al. 2019; Xie et al. 2017). The IoV and social Internet of Vehicles (SIoV) have set in motion a paradigm shift in vehicle technology and innovation (Contreras-Castillo et al. 2018; Jabri et al. 2019; Zia et al. 2020). An overview of the main components of the IoV, which is presented in Figure 1, includes vehicles with an on-board unit (OBU), a roadside unit (RSU), and cloud connectivity. VANETs and ITSs are looking forward to the implementation of the IoV (Alam et al. 2015) as an essential part of smart cities as a future vision of metropolitan areas (Contreras-Castillo et al. 2018; Fangchun et al. 2014). Vehicle manufacturers and smart city planners alike are looking forward to equipping their vehicles with smarter technology. Currently, implementation issues such as safety, policy, infrastructure standards, ethics, and specifications still exist, although the next wave of technology aims to implement vehicular networks either partially or fully.

The wireless nature of vehicular networks increases their vulnerability to threats (Alzamzami $\&$ Mahgoub 2020; Hasrouny et al. 2017). Malicious vehicles could divert traffic according to an organized strategy that may lead to disaster. A malicious activity would lead to traffic congestion and accidents, resulting in a possible risk to human life. The Internet connectivity to the 
80

81

82

83

84

85

86

87

88

89

90

91

92

93

94

95

96

97

98

99

100

101

102

103

104

105

106

107

108

109

110

111

112

113

114

115

116

117

118

119

vehicular network has increased the attack surface. The flexibility of vehicular networks needs to be such that they are able to accommodate vehicles that join and leave the network at any point in time (Patel \& Jhaveri 2015). These networks have no geographical limits because of their mobile characteristics. In this situation, vehicle-to-vehicle (V2V) communication is very significant. The availability of a centralized network is a common problem in VANETs, which means that vehicles may come under an unsupervised network at times. The disadvantage of a centralized network is the well-known single point of failure (SPoF) problem, where only a single point of disruption could lead to network unavailability. Previously (Fangchun et al. 2014), a broad overview of the IoV and related future challenges, such as communication, security, and dynamism, was presented. Another study discussed the basic visionary idea of the IoV (Mbelli \& Dwolatzky 2016), including potential benefits in coming years, and security was also considered as being an important concern for the future of the IoV. The development of the IoV greatly depends on the availability of intelligent systems for which there currently is a great need (Fangchun et al. 2014). VANETs present multidimensional security problems, and make security one of the basic challenges for ad hoc networks. The available trust evaluation and management solutions for VANETs / IoV need to be improved (Qiu et al. 2016; Sumithra \& Vadivel 2018; Yao et al. 2017).

Security, privacy, standards, and many other challenges would have to be overcome before a full-fledged vehicular network could be implemented (Lin et al. 2008). Vehicular networks possess unique characteristics in addition to those of many other types of networks, in that they require their own standards and protocols to manage the network. To overcome the problem of trust among the nodes in a vehicular network, researchers have proposed many models for trust evaluation in VANETs. Considering the security of a vehicular network, trust is one of the most important challenges (Hasrouny et al. 2017; Saini et al. 2015; Yao et al. 2017). Messages received by a node, such as information about accidents, traffic congestion, construction work in progress, natural disasters, and others, require the integrity of the information to be verified before action is taken. Vehicles with malicious intentions can divert traffic along specific routes. Many different techniques and methods have been adopted as approaches for trust evaluation over the years (Yao et al. 2017).

Over the years, several models for "vehicular network TM" have been proposed, and many researchers continue to work on trust models. Different techniques are being used to achieve the highest level of security using trust. As trust is a qualitative property, it is difficult to standardize. When the next generation of vehicular networks is implemented in the future, standardized TM would be necessary.

The purpose of our review is to conduct an in-depth exploration of the current models that have been presented to ensure that trust exists among the nodes of vehicular networks. Second, we aim to identify the strengths and weaknesses of existing models. Our final objective is to explore the potential implementation of context awareness in vehicular networks. The vehicle network is a dynamically changing network thus requires dynamic solutions. The dynamic system should be able to adapt according to the context to deal with the changes. Context 
120 awareness is one of the AI solutions that provides adaptivity to a system. Adaptivity is the

121 transforming ability to fit in a certain condition, Context awareness can enable adaptation [1].

122 As context awareness is an emerging area of artificial intelligence (AI), context awareness has

123 many advantages yet to be explored.

124 An adaptive mechanism needs to be used to ensure trust in a dynamic system (Yan et al.

125 2016). The importance of an environment-adaptive TM scheme for VANETs was discussed

126 (Pathan 2016) and it was concluded that the unwavering security infrastructure of VANETs lacks

127 the ability to meet the security requirements. The trust evaluation system must be flexible and

128 robust to adapt to different situations [2]. The TM deals with vast variable information, context

129 awareness is the way to use available information dependingon the situation, context awareness

130 does not rely on static system inputs. In a research study [3], authors discussed the need for

131 VANET TM schemes to be adaptive to the environment. A study on the implications of AI in

132 context awareness (Kofod-Petersen \& Cassens 2005) considered the usefulness of AI techniques

133 to build the context. We conclude our study by presenting researchers with advice to enable them

134 to solve different problems in vehicular networks.

\section{Trust}

136 Trustworthiness is the level of legitimacy, and trust can be evaluated against the data or node

137 in VANETs and ITS. Apart from securely delivering data between nodes, the integrity of the

138 received content is important (Ma et al. 2011; Yao et al. 2017; Zhou et al. 2015). Trust is an

139 important component of vehicular network security (Ma et al. 2011; Sumithra \& Vadivel 2018).

140 Other researchers (Ma et al. 2011; Saini et al. 2015; Yao et al. 2017) also discussed trust as a key

141 security challenge owing to the dynamic nature of a vehicular network. Trust could be static

142 where a trust level is assigned to certain types of vehicles/nodes such as RSUs or "police cars";

143 on the other hand, it could be dynamic where trust is developed based on interaction (Ma et al.

144 2011).

145 An objective of VANET security is to prevent messages from being forged during 146 communication (Hasrouny et al. 2017). In repute-based systems, it is difficult for a vehicle that

147 recently joined the network to prove its trustworthiness to the other nodes. In VANETs, the

148 trustworthiness of data is of greater significance than that of a node because of the nature of

149 these ad hoc networks (Hasrouny et al. 2017; Raw et al. 2013). Data-centric trust and

150 verification in V2V communication in VANETs need to be studied in more detail. Trust

151 evaluation techniques can be classified into three types (Hasrouny et al. 2017; Raya \& Hubaux

152 2007; Saini et al. 2015; Soleymani et al. 2017; Sumithra \& Vadivel 2018; Yao et al. 2017) as

153 shown in Figure 2. The trust model that evaluates trust based on the available information is data

154 centric. Entity centric trust model considers the trust associated with vehicle nodes.

\section{TM}

156 The responsibility of the TM system is to manage the real-time and long-term trust of the 157 nodes in a network. Second, to determine the legitimacy of the message received from different

158 nodes in the network. Most of the early TM models were based on data and entity now the trend

159 is moving towards hybrid TMs. The main problem areas include trust evaluation during fewer 
160 node availability, uninterrupted centralized connectivity, and dealing with the dynamic nature of

161 the network. Moreover, researchers are facing new challenges while developing TMs for IoV.

\section{Rationale for the review}

163 IoV is an emerging field that lacks standards and needs to be standardized before IoV is

164 implemented as part of smart cities. TM is one of the key components of IoV security.

165 Previously, several TM models for VANET / ITS were presented, and these studies serve as the

166 basis for IoV. In the absence of a comprehensive analytical study, this SLR provides a clear and

167 comprehensive overview of the available evidence. In addition, our work helps to identify

168 research gaps in our current understanding of the field. The objective of this review is to provide

169 a basis for the developers of TM models for IoV. Our findings laid the foundation for the

170 Intelligent TM System.

\section{Intended audience}

172 The article was intended to support academic and industry researchers working on TM in ad

173 hoc vehicle networks, in particular the emerging field IoV. The work is not limited to IoV, the

174 findings are equally beneficial for those working on IoT security. The open challenge sections

175 are specifically presented to researchers who are new to the field of vehicle communication and

176 need a strong research gap.

177

The remainder of the paper is organized as: section 2 presents the methodology of the systematic literature review (SLR), section 3 contains the results and discussion, and section 4 discusses the findings and challenges.

\section{Survey Methodology}

This research was conducted by using a method known as systemic literature review with the purpose of analyzing the limitations of this topic in a targeted way. Furthermore, we opted for meta-synthesis to help us identify problematic issues in current techniques and establish workable projections for further research in the area. The systematic study is based on existing PRISMA guidelines (Kitchenham 2004).

\section{Justification of SLR}

As discussed in the "existing review" section, few review studies have focused on TM in VANETs; furthermore, an SLR has not yet been carried out for the IoV. There has been a surge in SLRs that collect and review existing knowledge, to help new researchers entering this particular field of research.

2. Lack of focused studies

Most of the review studies on VANETs encompass the entire security paradigm, with TM included as a single section. Contrary to this, our study focuses entirely on TM in vehicular networks.

\section{Support related fields}


198

199

200

201

202

203

204

205

206

207

208

209

210

211

212

213

214

215

216

217

218

219

220

221

222

223

224

225

226

227

228

229

230

231

232

233

234

235

This SLR would be equally helpful for those interested in IoT security, especially for deviceto-device communication. Security has always been concerned with the IoT, many security threats emerge during IoT communication (Mollah et al. 2017) and trust is one of the solutions to ensure security (Jing et al. 2014). Second, the SLR is also intended to serve to apply context awareness to different types of ad hoc networks.

\section{Review protocol}

The review protocol for this study is based on the guidelines of a renowned review protocol PRISMA (Moher et al. 2009) and (Kitchenham 2004). We formulated the following protocol for this specific study as in Table 1.

\section{Objectives of the study}

Table 2 lists the research questions that cover the research objective of our SLR. The primary objective of this SLR is to analyze the core techniques that are used to manage trust among nodes in a vehicular network and motivated the construction of (RQ1): What are the current techniques for trust evaluation/management in the vehicular network? To identify the unique and useful aspects of each model (RQ2): "What improvements are being proposed for solving these problems?" To determine the weaknesses and strengths of each study, we highlight the gray areas of current models, which are expected to open new research dimensions. Finally, (RQ3) asks "Is context awareness suitable for the development of trust in a vehicular network?" We believe that the answers we provide to these questions culminate in a comprehensive study that not only offers new perspectives, but also opens new research dimensions.

\section{Selection criteria}

\section{First phase}

This study focuses on the existence of trust among nodes in vehicular networks. To formulate the search terms, we used different literature surveys on vehicular networks and searched online repositories. We initially started with the terms listed in the following section. A total of 256 articles were collected at the end of the first phase.

\section{Key terms}

Following key terms and search queries, searched for in different repositories. The key search terms are driven by research questions using PRISMA guidelines.

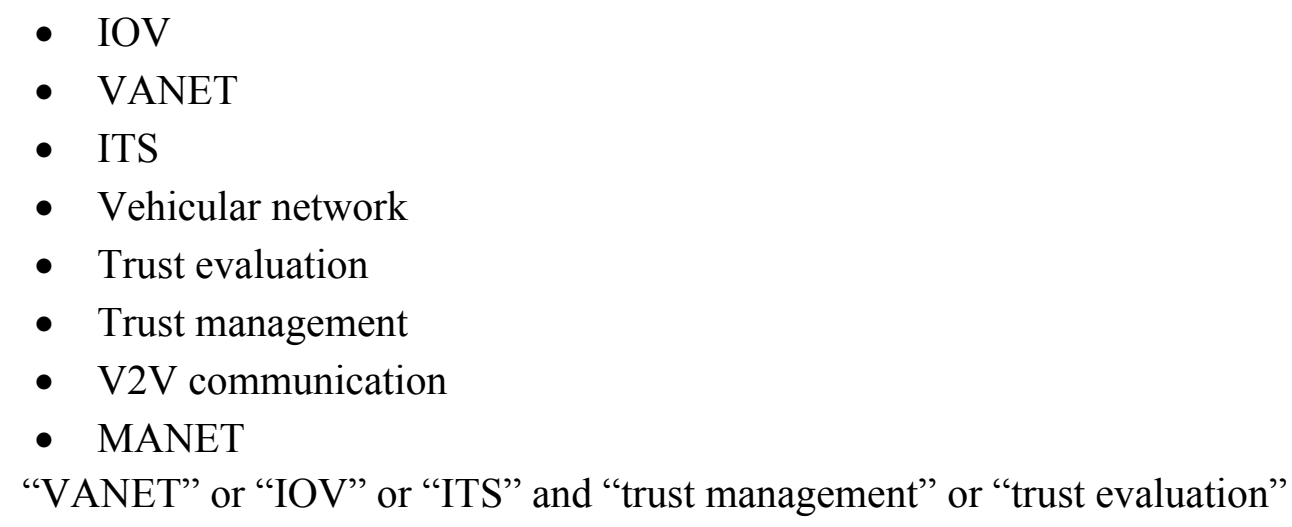


236

237

238

239

240

241

242

243

244

245

246

247

248

249

250

251

252

253

254

255

256

257

258

259

260

261

262

263

264

265

266

267

268

269

270

271

272

273

"VANET" or "IOV" and "trust management" and "V2V communication"

"MANET" and "trust management" or "evaluation and "context awareness"

"IOV" and "trust management" and "V2V communication"

"Internet of vehicles" and "trust evaluation" and "V2V communication"

"Context aware" and "VANET" and "trust management" or "trust evaluation"

\section{Second phase}

Because of the large number of articles that were retrieved in first phase, the second step involved tool-based filtering. Filtering by using the citation tool allowed us to identify duplicate studies from different sources and versions. At the end of this phase, we had a collection of 120 articles from different repositories.

\section{Third phase}

In this phase, the focus was "abstracts" other than the entire metadata. By considering the abstract of each paper, irrelevant data could be removed, 73 papers were excluded by this process which resulted in 47 articles remaining in our collection.

\section{Fourth phase}

Finally, all the remaining papers were read in detail. This process led to the exclusion of papers that did not meet the selection criteria or standard or were beyond the scope of our work. During this phase 16 articles were excluded, finally, 31 research articles were selected to conduct the study. The SLR selection process is illustrated in Figure 3.

\section{Scope of study}

Our study focuses on the following statement:

In-depth analysis of present trust evaluation and management in vehicular networks and implications of context awareness in trust establishment in vehicular networks.

\section{Limitations of study}

The following related areas are beyond the scope of our study:

- Routing protocols for the VANETs

- Cyber-attacks on VANETs

\section{Results and discussion}

The intense, in-depth study we conducted yielded significant results. In this section, we summarize our findings, followed by a discussion. All three of the research questions were used to analyze the papers with respect to the findings. Before the research questions are discussed, selected statistics are presented below. Finally, following a detailed analytical discussion, we present the open research challenges and directions to researchers in the field.

\section{Type of studies}

Table 3 presents the trends of studies conducted for trust evaluation and lists the year, category, and type of article ("I" for journal or "C" for conference). The studies were categorized as data-centric, entity-centric, or hybrid. Our final collection of selected papers contained 13 
274 "conference" articles and 19 "journal" articles. This number is based on the limitation of our

275

276

277

278

279

280

281

282

283

284

285

286

287

288

289

290

291

292

293

294

295

296

297

298

299

300

301

302

303

304

305

306

307

308

309

310

311

312

study, and might vary for any other study. Figure 4 shows the number of publications on TM that appeared during a certain decade. The Figure 4 also shows the number of papers excluded during the fourth phase while filtering the abstracts.

\section{Critical characteristics of trust models}

Table 4 presents certain important features of the trust model that were identified, i.e., the use of a central authority (CA), the authentication that was used, new node mechanism, uncertainty handling, and validation. These are a few of the most critical aspects of any TM system. A detailed discussion of the significance of these properties can be found in the discussion section.

\section{Simulation tool used in TMs}

Most of the papers contain a discussion of the simulator that was used in the particular study. These simulators are listed in Table 5. The purpose of specifying the simulator is to lay the ground for researchers to choose simulation tools for further study.

\section{Existing review studies}

To the best of our knowledge, a few review articles on trust evaluation and management have been reported. A few studies partially considered trust, but these reviews were not sufficiently comprehensive. The review studies in which TM in vehicular networks was discussed are presented in Table 6 (Arif et al. 2019; Kerrache et al. 2016; Soleymani et al. 2015; Sumithra \& Vadivel 2018). Other than focused studies, several review articles on VANET security included a discussion on trust. This includes (Sumithra \& Vadivel 2018) who, in their review study presented at a conference, discussed the fundamentals of VANETs and related security. Their focus was on fuzzy logic-based systems. Another conference presentation (Arif et al. 2019) on a survey of trust relating to VANETs, focused on secure routing and the quality of service in future technical and non-technical directions. A journal review article (Kerrache et al. 2016) was published on TM in VANETs, including a security overview, attacks, and available trust solutions. Another review study on TM specifically selected ten trust models and their performance was analyzed (Soleymani et al. 2015), the second focus of this study was the use of Fuzzy logic in TM.

Other than the above-mentioned focused studies, several reviews (Hasrouny et al. 2017; Raya \& Hubaux 2007; Saini et al. 2015) were conducted on ad hoc network security and VANETs. These broad studies paid slight attention to the significance of TM in VANETs.

\section{RQ1. What are the current techniques for trust evaluation/management in the vehicle network?}

\section{Approach by TMs}

The core approaches adapted by trust models are listed in Table 7, in which models that have certain key approaches in common are combined. By analyzing the data provided in Table 7, it is easy to identify the most and least commonly adapted approaches and the most common aspects 
313 of different models. The commonly employed approaches were extracted from Table 7 and are

314 plotted in Figure 5 for easy understandability. The bar graph in Figure 5 highlights the top ten

315 parameters used in different studies. Experience is the topmost property adapted by most of the

316 trust models, followed by neighbor-opinion and vehicles with special roles.

317 The core techniques, methodologies, and related components used by each TM model are

318 summarized in Table 8. As discussed in section 1, TM models can be categories as: data-centric,

319 entity-centric, and hybrid. Previously, researchers mostly focused on data-centric TMs, after

320 which they realized that node properties are an important part of any TM, resulting in the

321 proposal of many entity-based models. Hybrid models, are a combination of both of these

322 techniques. Other than these three categories, certain other characteristics play an important role

323 in TM, as discussed in this section. The aim of the discussion is to clarify the concepts behind

324 different techniques that are expected to lay the foundation for unification during the

325 standardization process. Furthermore, the discussion highlights certain aspects warranting further 326 research.

\section{Data-centric trust models}

These models are based on data (message) for trust evaluation. A data-centric model (Chen et al. 2010), with the aim of improving the scalability using trust, was proposed. The authors evaluated the trust by using an opinion-based approach to identify malicious nodes by evaluating the trust. In addition, they used a cluster and role-based approach with the majority opinion being one of the key elements of this approach.

A few similar models (Minhas et al. 2010b; Raya \& Hubaux 2007), in which a neighboring vehicle provides its opinion on the trustworthiness of events, were presented. The trust is computed on the basis of the highest vote. A drawback of these models is that messages can only be communicated among specified nodes in one group. These models can be used for distributed attacks using "neighbor node opinion." A second drawback of these models is that opinion calculation methods are not clearly defined.

Other researchers (Shaikh \& Alzahrani 2014) presented a decentralized TM system for VANETs. The scheme is based on different values calculated for the location and time to evaluate the trust. Their model seems effective in certain situations, but has limitations. Based on similarity, another trust model (Yang 2013) used the similarity between a node and the data to evaluate the trust level. Although a unique method of similarity was introduced, the technique was not validated. The trust was calculated based on direct and indirect experiences. The experimental details were not discussed, although the model seems to belong to the research field theoretically.

A model based on vehicle behavior and a Markov chain (Gazdar et al. 2012) was presented. The Markovian state transition was used to allocate and evaluate the trust of a node. In this model, all the vehicles act as monitors within their related perimeter. The model is distributed and maintains the record of trust locally. In another study (Wu et al. 2011). the researchers proposed an RSU-based data-centric trust model. An earlier data-centric trust model (Raya et al. 2008) considers role-based desperation, where nodes related to governmental organizations hold 
353 more trust credibility. In contrast, the model also considers the node trust level. Bayesian

354

355

356

357

358

359

360

361

362

363

364

365

366

367

368

369

370

371

372

373

374

375

376

377

378

379

380

381

382

383

384

385

386

387

388

389

390

391

392

inference and the Dempster-Shafer theory are used to handle uncertainty.

The data centric approach is used by earlier TMs, which lack the use of information associated with the node (entity). Entity centric and hybrid models have better capabilities than data centric models. It can be concluded from the above discussion that, even though the data centric approach is less effective, yet this approach is important for TM. It can play a key role in a hybrid TM system (Yao et al. 2017).

\section{Entity centric trust models}

These models are based on building trust against vehicle (entity). In a recently presented entity-centric trust model (Soleymani et al. 2017) the level of trust is assessed by fuzzy logic, using experience and probability. Authentication is based on the local ID and the location of the sender also plays a major role in the model. Another entity-centric distributed trust model (Haddadou et al. 2014) is based on the allocation of credit to the nodes used for TM. The model identifies the malicious node and motivates selfish nodes by awarding credits if they participate. The node is initially assigned a credit value, which increases or decreases over the time period.

A trust protocol based on a time frame (Ya et al. 2015) is presented with the aim of identifying malicious vehicles in the network. The trust value of a node is determined by the feedback from the neighboring nodes. A hybrid trust model that considered location privacy (Chen \& Wei 2013) was presented. The presented technique is based on beaconing, where the movement of nodes plays an important role. A combination of direct and indirect trust was used in this model to evaluate the trust, which was validated using Dempster-Shafer evidence. An ant colony-based cluster trust model was proposed (Sahoo et al. 2012). Clusters were formed by using different parameters such as direction and speed. The head of a cluster was selected based on a higher trust level. Trust was based on neighbor opinion.

A model based on prior experience, neighbor-suggestions, and CA was used to build node trust (Mármol \& Pérez 2012), which was achieved by using the reward and punishment method. The trust was determined in a fuzzy fashion to facilitate assessment by the system. Other entitycentric models (Cui et al. 2019; Li et al. 2013) used a mix of CA and cryptography. The primary problem with these models is that, once the vehicle has been authenticated, it cannot act maliciously. A decentralized agent-based trust model (Minhas et al. 2010a) using role, experience, and opinion, was presented. Another trust model (Biswas et al. 2011) was based on node-ID and local authentication by the public key, where digital signatures play a key role in this model for node identification. The assumption that "an authenticated node is trustable" is not realistic in VANETs. A scheme to ensure trust during communication in a VANET was proposed (Li et al. 2012). They set the "trust" as the reputation score of any node in the network. A message from highly reputed vehicles is considered trusted. However, experience is involved in building a reputation. Another unique aspect of their work is the feedback against the reporting vehicle.

An entity-centered framework for trust evaluation without a centralized system was proposed (Gazdar et al. 2018). The framework focuses on immediate knowledge between adjacent cars.

Peer] Comput. Sci. reviewing PDF | (CS-2020:07:50666:3:0:NEW 18 Oct 2020) 
393 Their system is also effective for fake message detection. The solution is based on the self-

394 organization of nodes in a network rather than depending on centralized authorities. The trust

395 level of a vehicle is evaluated using different parameters. Neighboring vehicles play an important

396 role in trust measurement. Based on the discussion and analysis of entity-centric techniques, it is

397 firmly established that these models are the core of any trust model. Without considering the

398 entity, the performance of trust models is compromised. On the other hand, simply relying on the 399 entity for trust evaluation is also not the ultimate solution.

400 By discussion and analysis of entity centric techniques, it is firmly established that these 401 models are the core of any trust models. Without considering entity the performance of trust 402 models is compromised. On the other hand, relying just on the entity for trust evaluation is also 403 not the ultimate solution.

\section{Hybrid trust models}

Most of the recent models are hybrid models, combining data and evaluate the legitimacy of the received message. A proposed hybrid trust approach (Yao et al. 2017) for VANETs is based on the assignment of classified weights to different entities of the system. The model applies experience and utility theory, and the authors assumed certain attributes and the pre-assignment of weight. A renowned group (Wei et al. 2014) applied a hybrid approach, based on a combination of Bayesian rules to find the trust and Dempster-Shafer theory for handling uncertainty. The approach is based on direct and indirect trust to calculate the overall trust. A hybrid framework for TM, based on neighbor nodes, was presented (Ahmed et al. 2017). The model divides trust into three categories: events, nodes, and recommendations. Based on consistency and similarity, the trust for a certain event is calculated and updated. The presence of legitimate events and nodes in the system is an assumption. In a long-term trust relationship model (Biswas et al. 2016) the main trust evaluation body is the RSU supported by CA at the top. The model assumes that all the vehicles in the networks are registered with the CA and maintain a certain level of trust.

A trust evaluation study based on cluster and intrusion detection is presented to deal with different attacks (Sedjelmaci \& Senouci 2015). The trust level is evaluated using majority voting by neighbor nodes. The model is subdivided into three: local, cluster head, and RSU. Trust is managed by the cluster head. The node is categorized in the list of trusted, suspicious, and attackers. The authors in (Rawat et al. 2015) worked on a combination of two different approaches, probabilistic and deterministic. The probability of a malicious node is calculated using Bayesian. The deterministic part calculates the trust message by calculating distances using signal strength, arrival time, and location. By most reliable nodes among adjacent cars (Abdelaziz et al. 2014), a new idea of filtering is used as a forwarding process. The implementation of the system avoids engaging in a network with cars recognized as likely dishonest users. A trust list is maintained locally by every vehicle, which is based on node interaction. Node velocity is used to determine the neighbors. The last message received serves as an integral part in evaluating trust since piggybacking is used. 
432

433

434

435

436

437

438

439

440

441

442

443

444

445

446

447

448

449

450

451

452

In a cluster-based trust framework, misbehaving vehicles are sorted by assessing their speed variation (Wahab et al. 2014). The system is based on a reward scheme for motivation. To counter the trust and untrusted nodes, the Dempster-Shafer module is set in place as a watchdog. The cluster head plays the main role in their model. The model seems very complicated and unrealistic in real situations. A study (Li et al. 2013) based on a centralized TM, all the nodes monitor neighboring nodes and forward their opinion to the centralized system. The central system finally calculates the trust value based on new and previous data. RSU also plays an important role in this system.

In a study (Zhang et al. 2013) researchers worked on a peer opinion based trust model, and peers after receiving a message provided their opinion and forwarded the message. The model combines many sub-modules, such as propagation, trust evaluation, and peer-to-peer trust modules. Finally, trust is monitored and managed by a CA. An RSU-managed trust model, using role and experience, was presented (Monir et al. 2013) and is unlike other models that associate the driver ID with trust evaluation. The trust level of a vehicle increases with time in terms of experience. A penalty for malicious nodes is also part of the system, which reduces the trust level of the node in the future.

Overall, hybrid models have more adaptability and dynamicity. As vehicular networks require dynamic solutions, it is essential to design a trust model in a hybrid manner. The hybrid models allow multiple dimensions and the properties of both data and nodes to be used for trust evaluation.

\section{RQ2. To what extent are the proposed models effective?}

In this section, we discuss the critical aspects of a trust model with respect to existing models. The purpose of the discussion is to facilitate the establishment of the effectiveness of the current models.

\section{Assumptions made by trust models}

Most models made certain assumptions about their TM processes that create inflexibility. In a dynamic environment, assumptions decrease the importance of the proposed solution. For instance, assumptions made by (Chen et al. 2010; Raya et al. 2008), special-vehicles are present in every situation, is unrealistic. Another assumption made by (Sedjelmaci \& Senouci 2015), "RSU is always available" which is practically unfeasible. Likewise, (Abdelaziz et al. 2014) assumes that all nodes are moving with the same velocity, which does not match the nature of the network. So much so, every model has some assumptions that make it hard to implement in realtime. The assumptions made by the trust models are listed in Table 9. However, assumptions regarding the implementation process prove to be a major hurdle. During a real traffic event, assuming the presence of special vehicles, RSU availability, pre-assigned trust, and many others can create serious problems while evaluating the trust. During a random event and network scenario, a trust model must not assume any variable to be completely implementable.

\section{Centralized or decentralized vehicular network}

There has always been a divide on whether ad hoc networks should be centralized or decentralized. Certain models (Abdelaziz et al. 2014; Gazdar et al. 2012; Haddadou et al. 2014; 
472 Saini et al. 2015; Shaikh \& Alzahrani 2014) considered decentralization as their approach,

473 whereas others applied a centralized approach (Hussain et al. 2016; Li et al. 2012; Li et al. 2013;

474 Mármol \& Pérez 2012; Monir et al. 2013). In a recent study (Arif et al. 2019) author

475 recommended centralized validation for TM. The main issue is the ultimate connectivity, which

476 is difficult to achieve in these networks. In addition, for security, centralized data storage and

477 management is essential. Although a practical solution would be to periodically synchronize trust

478 data with a centralized management system, completely relying on centralized management is

479 not the best practice. On the other hand, having a solely decentralized system causes a serious

480 threat to the ad hoc networks.

481

482

483

484

485

486

487

488

489

490

491

492

493

494

495

496

497

498

499

500

501

502

503

504

505

506

507

508

509

510

511

\section{Importance of authentication}

The first step in any security system is authentication with a vehicular network. Here, the point of dispute is "If the authentication alone fulfills the security requirements." A VANET is likely to experience a security breach after the legitimate approval of an authenticated node. In certain studies, authenticated users were assumed to always broadcast legitimate messages (Cui et al. 2019; Li et al. 2013; Mármol \& Pérez 2012). In a real situation, this may result in highly adverse security consequences, such as Sybil, DOS and DDOS, node-impersonation attacks and, message-tempering (Kerrache et al. 2016; Saini et al. 2015; Sakiz \& Sen 2017). Furthermore, (Sakiz \& Sen 2017) explains in their work that how an already authenticated node can be compromised by an attacker for blackhole and wormhole attacks. An authenticated node can be used for session hijacking, explains (Hasrouny et al. 2017).

Authentication must be dynamic and efficient in order to meet the requirements of IoT/IoV (Challa et al. 2020), for which researchers are working on lightweight authentication schemes (Vasudev et al. 2020). Authors in (Wazid et al. 2020) proposed a lightweight authentication scheme for IoT sensor data. Another research work (Wazid et al. 2018) on lightweight user authentication for internet of drones for direct communication is proposed. Blockchains can be a useful tool to build lightweight authentication solutions (Jangirala et al. 2019). The result shows that lightweight authentication schemes are suitable and secure for dynamic networks. Even though authentication is an essential aspect of an ad hoc network, we cannot rely solely on authentication.

\section{New node entering the network}

A critical issue during TM that is not taken into consideration is the entry of new nodes into the network. The question "how to assign a new node with initial trust" remains unanswered. Certain studies (Abdelaziz et al. ; Ahmad et al. 2018) assigned a new node a trust value of 1 or 0.5 , but the reason for this approach is not discussed in any of these papers. An intense study needs to be conducted to formalize the mechanism whereby a new node is introduced to a vehicular network.

\section{Cluster approach to handle trust}

A number of studies (Chen et al. 2010; Sahoo et al. 2012; Sedjelmaci \& Senouci 2015; Wahab et al. 2014) adopted a cluster approach for managing trust. It is observed in most of the studies that a cluster creates inflexibility in a network. A VANET has a dynamically changing 
512 topology (Xu et al. 2020), and inflexibility can cause the network to malfunction. Nonetheless,

513 this does not decrease the importance of clustering in certain scenarios. In conclusion, the cluster

514 approach can be used in TM but in limited scenarios. Cluster management needs to be defined

515 and standardized to be used successfully. Cluster head selection has also been discussed in

516 certain studies.

\section{Role-based approaches}

518 The presence of special vehicles (vehicles from governmental organizations such as

519 ambulances, police vehicles, or fire engines) in the network requires a role-based approach

520 (Ahmad et al. 2018; Alagar \& Wan 2015; Haddadou et al. 2014; Monir et al. 2013; Zhang et al.

521 2013). Role-based nodes are considered trustworthy in these models and are assigned a higher

522 trust level than other nodes. The question that remains unanswered is the approach to follow

523 when special vehicles are present in every event. Practically, it is not possible to have special

524 vehicles everywhere; eventually, the subtraction of role-based vehicles from the network at any

525 time would affect the performance of the trust model. Role-based nodes and their use have been

526 the topic of many studies, and this approach might be useful in certain situations.

\section{RSU-based approaches}

528 In the early phases of VANET research, RSUs became an essential part of the VANET

529 architecture (Behravesh \& Butler 2016). Models (Alagar \& Wan 2015; Biswas et al. 2011;

530 Biswas et al. 2016; Li et al. 2013; Monir et al. 2013; Sedjelmaci \& Senouci 2015; Wu et al.

531

532 2011) that used the RSU as the main authority for TM were proposed. A "trust value" was

533 assigned to an RSU depending on its location and coverage (Biswas et al. 2016). Another RSUbased study (Alagar \& Wan 2015) presented a trust scheme using role-based vehicles. Further,

534 they considered an RSU as a monitoring and managing node that also has a trust value, and the

535 overall network was controlled by the CA. A trust model involving RSU-based scheme (Wu et al. 2011) was proposed, in which an RSU receiving environmental change reports by all vehicles after receiving reports from the RSU is responsible for deciding whether any message is real or false. The feedback process serves to confirm the trustworthiness. However, relying on an RSU is not a practical approach, as RSUs are stationary nodes and RSU availability cannot be assured across the entire network (Chi et al. 2013; Haydari \& Yilmaz 2018). Despite all of these facts, the RSU continues to remain the main component of the VANET architecture and can be used wisely in certain situations or as a supporting node.

\section{Fuzzy logic in TM}

544

Fuzzy logic is a very useful tool and is used in many AI solutions. Two research groups (Mármol \& Pérez 2012; Soleymani et al. 2017) used fuzzy logic in their work to evaluate trust. According to the first group (Soleymani et al. 2017) reason for using fuzzy logic is the uncertainty within VANETs. Their results show that fuzzy logic can be used positively in trust evaluation. In a review study (Sumithra \& Vadivel 2018) author elaborated on the potential need for a fuzzy logic method for the trust model in VANETs. The study found coherence between VANET and fuzzy-based solutions. The author further mentioned that fuzzy approaches lack robustness and verity adoption, however, fuzzy methods seem to be a good solution if they are 
552 used in TM. The only disadvantage of fuzzy based TMs is the time consumed during the process 553 of computing the trust because a vehicular network cannot afford time delays.

554 Uncertainty handling while evaluating trust

555 A point of uncertainty always exists during trust evaluation (Yao et al. 2017). The majority of 556 models are unable to discuss the uncertainty in their solutions. A trust model without the ability 557 to handle uncertainty is considered an incomplete solution. Models that considered uncertainty 558 while evaluating trust (Chen \& Wei 2013; Haddadou et al. 2014; Monir et al. 2013; Wahab et al. 559 2014; Wei et al. 2014; Ya et al. 2015), processed the uncertainty by using probability approaches 560 such as Bayesian, DST, and Markov processes. Researchers designing a trust model need to 561 prioritize uncertainty handling.

\section{Use social media}

Social media could form a new addition to TM in IoV networks. TM in social vehicular networks was discussed on the basis of email and social media-based trust (Hussain et al. 2016). The model relies on CA to manage trust. The idea of considering social media was presented at a conference and seemed interesting, but is quite exposed to social media threats. Here it is important to note that the trust is associated with the user rather than the node. Driver-based trust faces the problem of disassociation, which might lead to miscalculation of the trust value. However, social media is an essential part of next-generation design (Zia et al. 2020).

\section{RQ3 Is context-awareness suitable for the development of trust in the vehicle network? \\ Context Awareness}

Vehicular networks are the most complex wireless networks and thus require flexibility in all aspects. Context awareness is an AI method that can be used to introduce flexibility to any system; contextual awareness is the ability to assess and adapt the environment (Ramos et al. 2008). Very few studies in this field have been reported, and a few studies have partly considered the context in vehicular networks (Ahmad et al. 2018; Alagar \& Wan 2015; Rostamzadeh et al. 2015). Our efforts to find the true essence of context awareness in these studies were unsuccessful. However, in related fields, such as distributed, ubiquitous, and mobile ad hoc networks, several studies related to context awareness have been reported.

A study of IoT architecture for smart cities (Gaur et al. 2015) used context awareness for scenario building. Their work focuses on the representation of knowledge of the smart city architecture through contextual awareness, which has become a necessity for ubiquitous systems (Taconet \& Kazi-Aoul 2010). Another study on interoperability in ubiquitous systems (Strang \& Linnhoff-Popien 2003) applied context awareness with positive results. A study on VANET/ITS (Al-Sultan et al. 2013) used context awareness to assess driver behavior. The outcome of the study was that context awareness has significant potential in relation to vehicular networks. The importance of context awareness in MANETs (Moloney \& Weber 2005) elaborated on the importance of context implementation in a system that is equipped with sensors. Moreover, the study suggested that any systems with sensor objects that can make good use of context awareness would be beneficial to vehicular networks while using node-to-node communication. 
592 Node-to-node context applications in MANETs were investigated (Wibisono et al. 2010) for the 593 purpose of context building in MANETs. Context-aware systems are able to adapt to changes 594 and increase the usability of any system and the efficacy thereof was discussed (Maneechai \& 595 Kamolphiwong 2014) in relation to distributed systems. In a futuristic study on different aspects 596 of vehicular network security (Wan et al. 2014), the use of context awareness in such systems is 597 strongly recommended, especially with respect to security and safety. Context awareness for 598 automatic TM is an important field of research (Yan et al. 2014).

A survey study (Yürür et al. 2016) on context awareness for the future of mobile systems, determined that a context-aware system has the ability to sense its environment. Furthermore, by using context the network increases its security, quality, and credibility. Their work concludes by highlighting the use of context for mobile-based systems. Furthermore, IoT architecture must be intelligent, and for that, context awareness is an effective method (Sarkar et al. 2014). The forthcoming networks are intelligent networks, for which context awareness is the key method to be adopted (Bilen \& Canberk 2015). A study on ambient computing (Babu \& Sivakumar 2014) discussed the ability of context awareness to enable adaptation for ubiquitous systems. Further, the researchers pointed out that a system that assesses the situation and acts accordingly must be equipped with context awareness. The challenge is to design context-based security middleware for a heterogeneous system. An earlier study determined that context awareness has special characteristics that support intelligent vehicle systems (Sørensen et al. 2004), but little work has been carried out in this direction. Further, they discuss the context that can be useful in V2V communication for intelligent vehicles.

Context awareness is a necessity for upcoming ad hoc networks, especially VANETs (Aguilar 614 et al. 2018). Their work on context modeling has significant potential in wireless networks.

615 Further, they indicated that the application of context to ad hoc networks may be advantageous.

616 Context awareness was employed to enhance the security of wireless networks (Lin et al. 2019).

617 This study was based on heterogeneous networks to show the implications of context awareness.

618 Their positive results showed that applying context awareness to security increased the 619 adaptivity.

In a VANET context-aware study for detecting misbehaving nodes (Chi et al. 2013), the authors applied context using neighboring nodes and used mobility information to construct the context. Their work yielded positive results that were the outcome of context awareness. A context knowledge representation scheme was presented (Ruta et al. 2018). Their work shows the implications of context in wireless networks, especially IoT-related technologies and VANETs, which strongly support our research question. In a recent study, context was employed to design a safety system in VANETs (Shen et al. 2016). The concept is for vehicles to share the road environment while driving safely. Routing can be improved by context awareness in wireless networks, especially in the IoT (Dhumane et al. 2018). A context-based VANET privacy model (Emara et al. 2015) that implemented contextual privacy successfully improved location tracing. The work was evaluated against the quality of service and found to be satisfactory even after the application of context awareness. 
632

633

634

635

636

637

638

639

640

641

642

643

644

645

646

647

648

649

650

651

652

653

654

655

656

657

658

659

660

661

662

663

664

665

666

667

668

669

Based on the above discussion and relevant studies, it is established that context awareness has the potential to be applied to vehicular networks for TM. Nonetheless, the term context could be interpreted to have a general meaning and many related challenges would need to be overcome. As the context has already been considered for MANETs, it could be used as the foundation for VANET/IoV as well. A comparative overview is presented in Table 10 based on a published report (Sharma \& Kaushik 2019).

\section{Summary at a glance}

An overview of the different technologies, the extent to which they overlap, related issues, and the potential future of these technologies is presented in Figure 6. Furthermore, Table 11 summarizes the major properties of the trust model. Only journal articles were considered, the reason for the exclusion of the conference papers at this point is to be precise, second, they are unable to provide detailed information on methodology, experimental results and analysis). The aim of the summary is to produce a comprehensive yet brief description for researchers in the field. According to the statistics in Table 11, two very important aspects of the trust model have been ignored by most of the researchers i.e., hopping and beaconing. Most malicious activities occur during hopping and beaconing (Jang et al. 2018; Suman et al. 2017).

RSUs, an important component of the VANET infrastructure, were not considered in any of the studies. However, RSUs were included in most of the conference idea presentations. As an RSU is an important entity with many functionalities, it can be used to take maximum advantage of the resources.

Analyzing the statistics in Table 11 shows that TM is a significant problem. Most studies have ignored certain major aspects. Therefore, dynamic trust models that consider maximum aspects to attain the highest level of trust are in demand. The inclusion of all aspects in a single trust model can be challenging, therefore requires an adaptive solution. Context awareness is a method that provides flexible adaptation to a complex system (Ramos et al. 2008).

\section{Open challenges and future research directions}

Based on the analysis and observations of this study, the following are open research challenges that need to be addressed. This is expected to provide research directions for researchers interested in this field, Table 12 provides research challenges and directions related to identified gray areas. Furthermore, the future vision of IoV is discussed to provide clear goals, helping researchers to work on a broader canvas.

1) Designing intelligent TM frameworks for vehicular networks. From results, it can be inferred that TM is a complex problem thus requires Intelligent systems. This will enhance the overall performance of the TM.

2) Choosing between a centralized or decentralized approach has always been debatable. A comparative analysis of centralized and decentralized approaches for vehicular ad hoc networks is required. This analysis will provide clear benefits and drawbacks to each approach. 
670 3) Nature-inspired computing has similarities with ad hoc vehicular networks. Detailed

671

672

673

674

675

676

677

678

679

680

681

682

683

684

685

686

687

688

689

690

691

692

693

694

695

696

697

698

699

700

701

702

703

704

705

706

707

708

709 analytical study on the coherence of swarm computing with vehicular networks is required. Swarm computing, with its intelligent capabilities, has some characteristics similar to the vehicle network. Some research work involving ant-colony is conducted in this field.

4) Solving the "node initialization" problem for TM. Whenever a new node joins an ad hoc network different initial trust credits need to be allocated. The trust value allocation to a new node in a vehicular network is one of the challenging problems for trust models.

5) Cluster management for vehicular network trust models. The studies using cluster have no common approach for managing the trust. An in-depth study is required to analyze and provide standards. A cluster approach can also be used as a subpart of the network security solution.

6) Overcoming uncertainty while evaluating trust in VANETs. Uncertainty is one of the complex problems in trust evaluation, comprehensive solutions are required in this area to handle uncertainty. A few works have been carried out in this aspect of TM models.

7) Use of social media for security in vehicular networks. Social media has become a part of our lives, this research area needs further exploration for security implementation. Some works have been carried out in this area showing positive results.

8) Designing IoV cyber security protocols. IoV is exposed to cyber-attacks due to internet connectivity. It, therefore, requires standard security protocols, specially designed for IoV cyber-security.

9) Using context awareness for security in ad hoc networks. Context awareness provides intelligent solutions, existing work in this field shows positive results. Coherence between both fields needs to be further investigated.

10) Context knowledge representation methods for vehicular networks. Knowledge representation is one of the complex problems faced while applying context awareness. Before implementing the context awareness research needs to work out the knowledge representation methods.

11) Context awareness in the vehicular environment. Within this area some research work is carried out which shows promising results, further exploration is needed for better solutions.

\section{Framing the future vision of loV, and recommendations}

$\mathrm{IoV}$ is expected to become part of the future smart city and intelligent transport system, the vision of future $\mathrm{IoV}$ is set out in the following key points and provides researches on a broadspectrum expectation.

1) Soon, IoV is expected to be fully or partially implemented as part of smart cities.

2) Conventional security is expected to be replaced with Intelligent security solutions for vehicular networks.

3) Trust between nodes is expected to be a standard part of future vehicular network security, there is still a potential gap in this direction.

4) Fog and edge technology are also making way for vehicular networks that will be a good addition to the IoV. 
710

711

712

713

714

715

716

717

718

719

720

721

722

723

724

725

726

727

728

729

730

731

732

733

734

735

736

737

738

739

740

741

742

743

744

745

746

747

748

749

750

5) Vehicular communication generates huge data in seconds, the Big Data has a huge potential in vehicular networks, yet to be explored. Driver behavior, traffic patterns, accident prevention, smooth traffic flow, and many other interesting discoveries and solutions can be made using Big Data analysis.

6) Context awareness has an enormous potential in the vehicular network yet to be explored and implemented in the future.

7) A mix of a centralized and decentralized network is also expected to be established.

8) Another aspect that is worthy of further investigation is the management of cloud resources during vehicular communication. Various problems arise with regard to the nature of the communication.

9) The social network seems to be part of IoV. Apart from the human social network, the social network of vehicles is also likely to be developed.

\section{Conclusion}

The IoV is a potential part of the intelligent transport solution for the next wave of smart cities. As the IoV is still in the development phase, applicable standards need to be developed and recognized worldwide. Thus, the trust models in the IoV are yet to be standardized and transformed from VANETs and the ITS. We devoted our efforts to produce a systematic review in which we examined all available trust models. The outcomes of the meta-analysis highlighted all dimensions, including gray areas. Further, to fulfill the need for intelligent solutions, we tried to establish the potential benefits of context awareness in vehicular networks, which would not only facilitate intelligent TM but also address other security issues. Our work could be useful to develop standards for TM in vehicular networks. The results of the study are equally helpful for designing IoT security solutions, as device-to-device communication is a key aspect of the future IoT.

In terms of future research, we raised open questions for researchers in the Open Challenges section. Answering each question is likely to require intense research efforts. The outcome of our work is expected to be equally fruitful for other IoT solutions, ad hoc networks, and trust-related studies.

\section{Acknowledgments:}

\section{References}

Abdelaziz KC, Lagraa N, and Lakas A. 2014. Trust model with delayed verification for message relay in VANETs. 2014 International Wireless Communications and Mobile Computing Conference (IWCMC): IEEE. p 700-705.

Aguilar J, Jerez M, and Rodríguez T. 2018. CAMeOnto: Context awareness meta ontology modeling. Applied computing and informatics 14:202-213.

Ahmad F, Franqueira VN, and Adnane A. 2018. TEAM: A trust evaluation and management framework in context-enabled vehicular ad-hoc networks. IEEE access 6:28643-28660.

Ahmed S, Al-Rubeaai S, and Tepe K. 2017. Novel trust framework for vehicular networks. IEEE Transactions on Vehicular Technology 66:9498-9511. 
751

752

753

754

755

756

757

758

759

760

761

762

763

764

765

766

767

768

769

770

771

772

773

774

775

776

777

778

779

780

781

782

783

784

785

786

787

788

789

790

791

792

793

794

795

796

797

798

799

800

801

Al-Sultan S, Al-Bayatti AH, and Zedan H. 2013. Context-aware driver behavior detection system in intelligent transportation systems. IEEE Transactions on Vehicular Technology 62:4264-4275.

Alagar V, and Wan K. 2015. Context-Aware Trust-Based Management of Vehicular Ad-Hoc Networks (VANETs). 2015 IEEE 12th Intl Conf on Ubiquitous Intelligence and Computing and 2015 IEEE 12th Intl Conf on Autonomic and Trusted Computing and 2015 IEEE 15th Intl Conf on Scalable Computing and Communications and Its Associated Workshops (UIC-ATC-ScalCom): IEEE. p 255-261.

Alam KM, Saini M, and El Saddik A. 2015. Toward social internet of vehicles: Concept, architecture, and applications. IEEE access 3:343-357.

Alzamzami O, and Mahgoub I. 2020. Link Utility Aware Geographic Routing for Urban VANETs using Two-Hop Neighbor Information. Ad Hoc Networks:102213.

Arif M, Wang G, Bhuiyan MZA, Wang T, and Chen J. 2019. A survey on security attacks in VANETs: communication, applications and challenges. Vehicular Communications: 100179.

Babu AKA, and Sivakumar R. 2014. Development of ontology based middleware for context awareness in ambient intelligence. 2014 14th International Conference on Hybrid Intelligent Systems: IEEE. p 219-224.

Behravesh E, and Butler A. 2016. Evaluation of the IEEE 802.11p multi-channel operation in vehicular networks. PeerJ Preprints 4:e2633v1. https://doi.org/10.7287/peerj.preprints.2633v1

Bilen T, and Canberk B. 2015. Binary context tree based middleware for next generation context aware networks. 2015 3rd International Conference on Future Internet of Things and Cloud: IEEE. p 93-99.

Biswas S, Misic J, and Misic V. 2011. ID-based safety message authentication for security and trust in vehicular networks. 201131 st international conference on distributed computing systems workshops: IEEE. p 323-331.

Biswas T, Sanzgiri A, and Upadhyaya S. 2016. Building long term trust in vehicular networks. 2016 IEEE 83rd Vehicular Technology Conference (VTC Spring): IEEE. p 1-5.

Challa S, Das AK, Gope P, Kumar N, Wu F, and Vasilakos AV. 2020. Design and analysis of authenticated key agreement scheme in cloud-assisted cyber-physical systems. Future Generation Computer Systems 108:1267-1286.

Chen C, Zhang J, Cohen R, and Ho P-H. 2010. A trust-based message propagation and evaluation framework in vanets. Proceedings of the Int Conf on Information Technology Convergence and Services.

Chen Y-M, and Wei Y-C. 2013. A beacon-based trust management system for enhancing user centric location privacy in VANETs. Journal of Communications and Networks 15:153163.

Chi J, Jo Y, Park H, and Park S. 2013. Intersection-priority based optimal RSU allocation for VANET. 2013 Fifth International Conference on Ubiquitous and Future Networks (ICUFN): IEEE. p 350-355.

Contreras-Castillo J, Zeadally S, and Guerrero-Ibañez JA. 2018. Internet of vehicles: Architecture, protocols, and security. IEEE Internet of Things Journal 5:3701-3709.

Cui J, Wu D, Zhang J, Xu Y, and Zhong H. 2019. An Efficient Authentication Scheme Based on Semi-trusted Authority in VANETs. IEEE Transactions on Vehicular Technology.

Dhumane A, Guja S, Deo S, and Prasad R. 2018. Context Awareness in loT Routing. 2018 Fourth International Conference on Computing Communication Control and Automation (ICCUBEA): IEEE. p 1-5.

Emara K, Woerndl W, and Schlichter J. 2015. CAPS: Context-aware privacy scheme for VANET safety applications. Proceedings of the 8th ACM conference on security \& privacy in wireless and mobile networks: ACM. p 21.

Peer] Comput. Sci. reviewing PDF | (CS-2020:07:50666:3:0:NEW 18 Oct 2020) 
802 Fangchun Y, Shangguang W, Jinglin L, Zhihan L, and Qibo S. 2014. An overview of internet of

803

804

805

806

807

808

809

810

811

812

813

814

815

816

817

818

819

820

821

822

823

824

825

826

827

828

829

830

831

832

833

834

835

836

837

838

839

840

841

842

843

844

845

846

847

848

849

850

851 vehicles. China Communications 11:1-15.

Gaur A, Scotney B, Parr G, and McClean S. 2015. Smart city architecture and its applications based on loT. Procedia Computer Science 52:1089-1094.

Gazdar T, Belghith A, and Abutair H. 2018. An enhanced distributed trust computing protocol for VANETs. IEEE access 6:380-392.

Gazdar T, Rachedi A, Benslimane A, and Belghith A. 2012. A distributed advanced analytical trust model for VANETs. 2012 IEEE Global Communications Conference (GLOBECOM): IEEE. p 201-206.

Haddadou N, Rachedi A, and Ghamri-Doudane Y. 2014. A job market signaling scheme for incentive and trust management in vehicular ad hoc networks. IEEE Transactions on Vehicular Technology 64:3657-3674.

Hamid UZA, Zamzuri H, and Limbu DK. 2019. Internet of vehicle (loV) applications in expediting the implementation of smart highway of autonomous vehicle: A survey. Performability in Internet of Things: Springer, 137-157.

Hasrouny H, Samhat AE, Bassil C, and Laouiti A. 2017. VANet security challenges and solutions: A survey. Vehicular Communications 7:7-20.

Haydari A, and Yilmaz Y. 2018. Real-time detection and mitigation of ddos attacks in intelligent transportation systems. 2018 21st International Conference on Intelligent Transportation Systems (ITSC): IEEE. p 157-163.

Hussain R, Nawaz W, Lee J, Son J, and Seo JT. 2016. A hybrid trust management framework for vehicular social networks. International Conference on Computational Social Networks: Springer. p 214-225.

Jabri I, Mekki T, Rachedi A, and Jemaa MB. 2019. Vehicular fog gateways selection on the internet of vehicles: A fuzzy logic with ant colony optimization based approach. Ad Hoc Networks 91:101879.

Jang J, Kirat DH, Kwon BJ, Schales DL, and Stoecklin MP. 2018. Detecting malicious beaconing communities using lockstep detection and co-occurrence graph. In: Patents G, editor. US Patent Application No 15/626,767. inventors; International Business Machines Corp, U.S. Patent Application No. 15/626,767, USA: Google Patents.

Jangirala S, Das AK, and Vasilakos AV. 2019. Designing secure lightweight blockchain-enabled RFID-based authentication protocol for supply chains in $5 \mathrm{G}$ mobile edge computing environment. IEEE Transactions on Industrial Informatics.

Jing Q, Vasilakos AV, Wan J, Lu J, and Qiu DJWN. 2014. Security of the Internet of Things: perspectives and challenges. 20:2481-2501.

Kerrache CA, Calafate CT, Cano J-C, Lagraa N, and Manzoni P. 2016. Trust management for vehicular networks: An adversary-oriented overview. IEEE access 4:9293-9307.

Kitchenham B. 2004. Procedures for performing systematic reviews. Keele, UK, Keele University 33:1-26.

Kofod-Petersen A, and Cassens J. 2005. Using activity theory to model context awareness. International Workshop on Modeling and Retrieval of Context: Springer. p 1-17.

Li Q, Malip A, Martin KM, Ng S-L, and Zhang J. 2012. A reputation-based announcement scheme for VANETs. IEEE Transactions on Vehicular Technology 61:4095-4108.

Li X, Liu J, Li X, and Sun W. 2013. RGTE: A reputation-based global trust establishment in VANETs. 2013 5th International Conference on Intelligent Networking and Collaborative Systems: IEEE. p 210-214.

Lin H, Yan Z, and Fu Y. 2019. Adaptive security-related data collection with context awareness. Journal of network and computer applications 126:88-103.

Lin X, Lu R, Zhang C, Zhu H, Ho P-H, and Shen X. 2008. Security in vehicular ad hoc networks. IEEE communications magazine 46:88-95. 
852

853

854

855

856

857

858

859

860

861

862

863

864

865

866

867

868

869

870

871

872

873

874

875

876

877

878

879

880

881

882

883

884

885

886

887

888

889

890

891

892

893

894

895

896

897

898

899

900

901

Ma S, Wolfson O, and Lin J. 2011. A survey on trust management for intelligent transportation system. Proceedings of the 4th ACM SIGSPATIAL International Workshop on Computational Transportation Science: ACM. p 18-23.

Maneechai S, and Kamolphiwong S. 2014. Modeling design of the distributed reasoning based on Chord. 2014 11th International Conference on Electrical Engineering/Electronics, Computer, Telecommunications and Information Technology (ECTI-CON): IEEE. p 1-6.

Mármol FG, and Pérez GM. 2012. TRIP, a trust and reputation infrastructure-based proposal for vehicular ad hoc networks. Journal of network and computer applications 35:934-941.

Mbelli TM, and Dwolatzky B. 2016. Cyber security, a threat to cyber banking in South Africa: An approach to network and application security. Cyber Security and Cloud Computing (CSCloud), 2016 IEEE 3rd International Conference on: IEEE. p 1-6.

Minhas UF, Zhang J, Tran T, and Cohen R. 2010a. A multifaceted approach to modeling agent trust for effective communication in the application of mobile ad hoc vehicular networks. IEEE Transactions on Systems, Man, and Cybernetics, Part C (Applications and Reviews) 41:407-420.

Minhas UF, Zhang J, Tran T, and Cohen R. 2010b. Towards expanded trust management for agents in vehicular ad-hoc networks. International Journal of Computational Intelligence: Theory and Practice (IJC/TP) 5:03-15.

Moher D, Liberati A, Tetzlaff J, and Altman DG. 2009. Preferred reporting items for systematic reviews and meta-analyses: the PRISMA statement. Annals of internal medicine 151:264269.

Mollah MB, Azad MAK, and Vasilakos AV. 2017. Secure data sharing and searching at the edge of cloud-assisted internet of things. IEEE Cloud Computing 4:34-42.

Moloney M, and Weber S. 2005. A context-aware trust-based security system for ad hoc networks. Workshop of the 1st International Conference on Security and Privacy for Emerging Areas in Communication Networks, 2005: IEEE. p 153-160.

Monir M, Abdel-Hamid A, and El Aziz MA. 2013. A categorized trust-based message reporting scheme for VANETs. International Conference on Security of Information and Communication Networks: Springer. p 65-83.

Patel NJ, and Jhaveri RH. 2015. Trust based approaches for secure routing in VANET: a survey. Procedia Computer Science 45:592-601.

Pathan A-SK. 2016. Security of self-organizing networks: MANET, WSN, WMN, VANET: CRC press.

Qiu T, Luo D, Xia F, Deonauth N, Si W, and Tolba A. 2016. A greedy model with small world for improving the robustness of heterogeneous Internet of Things. Computer Networks 101:127-143.

Ramos C, Augusto JC, and Shapiro D. 2008. Ambient intelligence-the next step for artificial intelligence. IEEE Intelligent Systems 23:15-18.

Raw RS, Kumar M, and Singh N. 2013. Security challenges, issues and their solutions for VANET. International journal of network security \& its applications 5:95.

Rawat DB, Yan G, Bista BB, and Weigle MC. 2015. Trust On the Security of Wireless Vehicular Ad-hoc Networking. Ad Hoc \& Sensor Wireless Networks 24:283-305.

Raya M, and Hubaux J-P. 2007. Securing vehicular ad hoc networks. Journal of computer security 15:39-68.

Raya M, Papadimitratos P, Gligor VD, and Hubaux J-P. 2008. On data-centric trust establishment in ephemeral ad hoc networks. IEEE INFOCOM 2008-The 27th Conference on Computer Communications: IEEE. p 1238-1246.

Rostamzadeh K, Nicanfar H, Torabi N, Gopalakrishnan S, and Leung VCM. 2015. A ContextAware Trust-Based Information Dissemination Framework for Vehicular Networks. IEEE Internet of Things Journal 2:121-132. 10.1109/JIOT.2015.2388581

Peer] Comput. Sci. reviewing PDF | (CS-2020:07:50666:3:0:NEW 18 Oct 2020) 
902

903

904

905

906

907

908

909

910

911

912

913

914

915

916

917

918

919

920

921

922

923

924

925

926

927

928

929

930

931

932

933

934

935

936

937

938

939

940

941

942

943

944

945

946

947

948

949

950

951

Ruta M, Scioscia F, Gramegna F, leva S, Di Sciascio E, and De Vera RP. 2018. A knowledge fusion approach for context awareness in vehicular networks. IEEE Internet of Things Journal 5:2407-2419.

Sahoo RR, Panda R, Behera DK, and Naskar MK. 2012. A trust based clustering with Ant Colony Routing in VANET. 2012 Third International Conference on Computing, Communication and Networking Technologies (ICCCNT'12): IEEE. p 1-8.

Saini M, Alelaiwi A, and Saddik AE. 2015. How close are we to realizing a pragmatic VANET solution? A meta-survey. ACM Computing Surveys (CSUR) 48:29.

Sakiz F, and Sen S. 2017. A survey of attacks and detection mechanisms on intelligent transportation systems: VANETs and loV. Ad Hoc Networks 61:33-50.

Sarkar C, SN AUN, Prasad RV, Rahim A, Neisse R, and Baldini G. 2014. DIAT: A scalable distributed architecture for loT. IEEE Internet of Things Journal 2:230-239.

Sedjelmaci H, and Senouci SM. 2015. An accurate and efficient collaborative intrusion detection framework to secure vehicular networks. Computers \& Electrical Engineering 43:33-47.

Shaikh RA, and Alzahrani AS. 2014. Intrusion-aware trust model for vehicular ad hoc networks. Security and communication networks 7:1652-1669.

Sharma S, and Kaushik B. 2019. A survey on internet of vehicles: Applications, security issues \& solutions. Vehicular Communications 20:100182.

Shen Y, Jeong J, Oh T, and Son SH. 2016. CASD: a framework of context-awareness safety driving in vehicular networks. 2016 30th International Conference on Advanced Information Networking and Applications Workshops (WAINA): IEEE. p 252-257.

Soleymani SA, Abdullah AH, Hassan WH, Anisi MH, Goudarzi S, Baee MAR, and Mandala S. 2015. Trust management in vehicular ad hoc network: a systematic review. EURASIP Journal on Wireless Communications and Networking 2015:146.

Soleymani SA, Abdullah AH, Zareei M, Anisi MH, Vargas-Rosales C, Khan MK, and Goudarzi S. 2017. A secure trust model based on fuzzy logic in vehicular ad hoc networks with fog computing. IEEE access 5:15619-15629.

Sørensen C-F, Wu M, Sivaharan T, Blair GS, Okanda P, Friday A, and Duran-Limon H. 2004. A context-aware middleware for applications in mobile ad hoc environments. Proceedings of the 2nd workshop on Middleware for pervasive and ad-hoc computing: ACM. p 107110.

Strang T, and Linnhoff-Popien C. 2003. Service interoperability on context level in ubiquitous computing environments. Intl Conf on Advances in Infrastructure for Electronic Business, Education, Science, Medicine, and Mobile Technologies on the Internet (SSGRR2003w): Citeseer.

Suman N, Srinivas K, and Rao S. 2017. Malicious Detection of Packet Dropping in Wireless AdHoc Network.

Sumithra S, and Vadivel R. 2018. An Overview of Various Trust Models for VANET Security Establishment. 2018 9th International Conference on Computing, Communication and Networking Technologies (ICCCNT): IEEE. p 1-7.

Taconet C, and Kazi-Aoul Z. 2010. Building Context-Awareness Models for Mobile Applications. JDIM 8:78-87.

Vasudev H, Das D, Vasilakos AV, and Engineering E. 2020. Secure message propagation protocols for loVs communication components. Computers \& Electrical Engineering 82:106555.

Wahab OA, Otrok H, and Mourad A. 2014. A cooperative watchdog model based on DempsterShafer for detecting misbehaving vehicles. Computer Communications 41:43-54.

Wan J, Zhang D, Zhao S, Yang LT, and Lloret J. 2014. Context-aware vehicular cyber-physical systems with cloud support: architecture, challenges, and solutions. IEEE communications magazine 52:106-113.

Peer] Comput. Sci. reviewing PDF | (CS-2020:07:50666:3:0:NEW 18 Oct 2020) 
952

953

954

955

956

957

958

959

960

961

962

963

964

965

966

967

968

969

970

971

972

973

974

975

976

977

978

979

980

981

982

983

984

985

986

987

988

989

990

991

992
Wazid M, Das AK, Bhat V, and Vasilakos AV. 2020. LAM-CloT: Lightweight authentication mechanism in cloud-based IoT environment. Journal of network and computer applications 150:102496.

Wazid M, Das AK, Kumar N, Vasilakos AV, and Rodrigues JJ. 2018. Design and analysis of secure lightweight remote user authentication and key agreement scheme in Internet of drones deployment. IEEE Internet of Things Journal 6:3572-3584.

Wei Z, Yu FR, and Boukerche A. 2014. Trust based security enhancements for vehicular ad hocnetworks. Proceedings of the fourth ACM international symposium on Development and analysis of intelligent vehicular networks and applications: ACM. p 103-109.

Wibisono W, Zaslavsky A, and Ling S. 2010. Comihoc: A middleware framework for context management in manet environment. 2010 24th IEEE International Conference on Advanced Information Networking and Applications: IEEE. p 620-627.

Wu A, Ma J, and Zhang S. 2011. RATE: a RSU-aided scheme for data-centric trust establishment in VANETs. 2011 7th International Conference on Wireless Communications, Networking and Mobile Computing: IEEE. p 1-6.

Xie Y, Su X, He Y, Chen X, Cai G, Xu B, and Ye W. 2017. Stm32-based vehicle data acquisition system for internet-of-vehicles. Computer and Information Science (ICIS), 2017 IEEE/ACIS 16th International Conference on: IEEE. p 895-898.

Xu Y, Tong S, Zhang T, Sun W, Hu X, and Xiang Q. 2020. COMPASS: Directing Named Data Transmission in VANETs by Dynamic Directional Interfaces. IEEE access 8:8418-8435.

Ya X, Shihui Z, and Bin S. 2015. Trusted GPSR protocol without reputation faking in VANET. The Journal of China Universities of Posts and Telecommunications 22:22-55.

Yan Z, Zhang P, and Vasilakos AV. 2014. A survey on trust management for Internet of Things. Journal of network and computer applications 42:120-134.

Yan Z, Zhang P, and Vasilakos AV. 2016. A security and trust framework for virtualized networks and software-defined networking. Security communication networks 9:3059-3069.

Yang N. 2013. A similarity based trust and reputation management framework for vanets. International Journal of Future Generation Communication and Networking 6:25-34.

Yao X, Zhang X, Ning H, and Li P. 2017. Using trust model to ensure reliable data acquisition in VANETs. Ad Hoc Networks 55:107-118.

Yürür Ö, Liu CH, Sheng Z, Leung VC, Moreno W, and Leung KK. 2016. Context-awareness for mobile sensing: A survey and future directions. IEEE Communications Surveys \& Tutorials 18:68-93.

Zhang J, Chen C, and Cohen R. 2013. Trust modeling for message relay control and local action decision making in VANETs. Security and communication networks 6:1-14.

Zhou J, Dong X, Cao Z, and Vasilakos AV. 2015. Secure and privacy preserving protocol for cloud-based vehicular DTNs. IEEE Transactions on Information Forensics Security and communication networks 10:1299-1314.

Zia K, Shafi M, and Farooq U. 2020. Improving Recommendation Accuracy Using Social Network of Owners in Social Internet of Vehicles. Future Internet 12:69. 


\section{Figure 1}

An overview of the main components of the loV

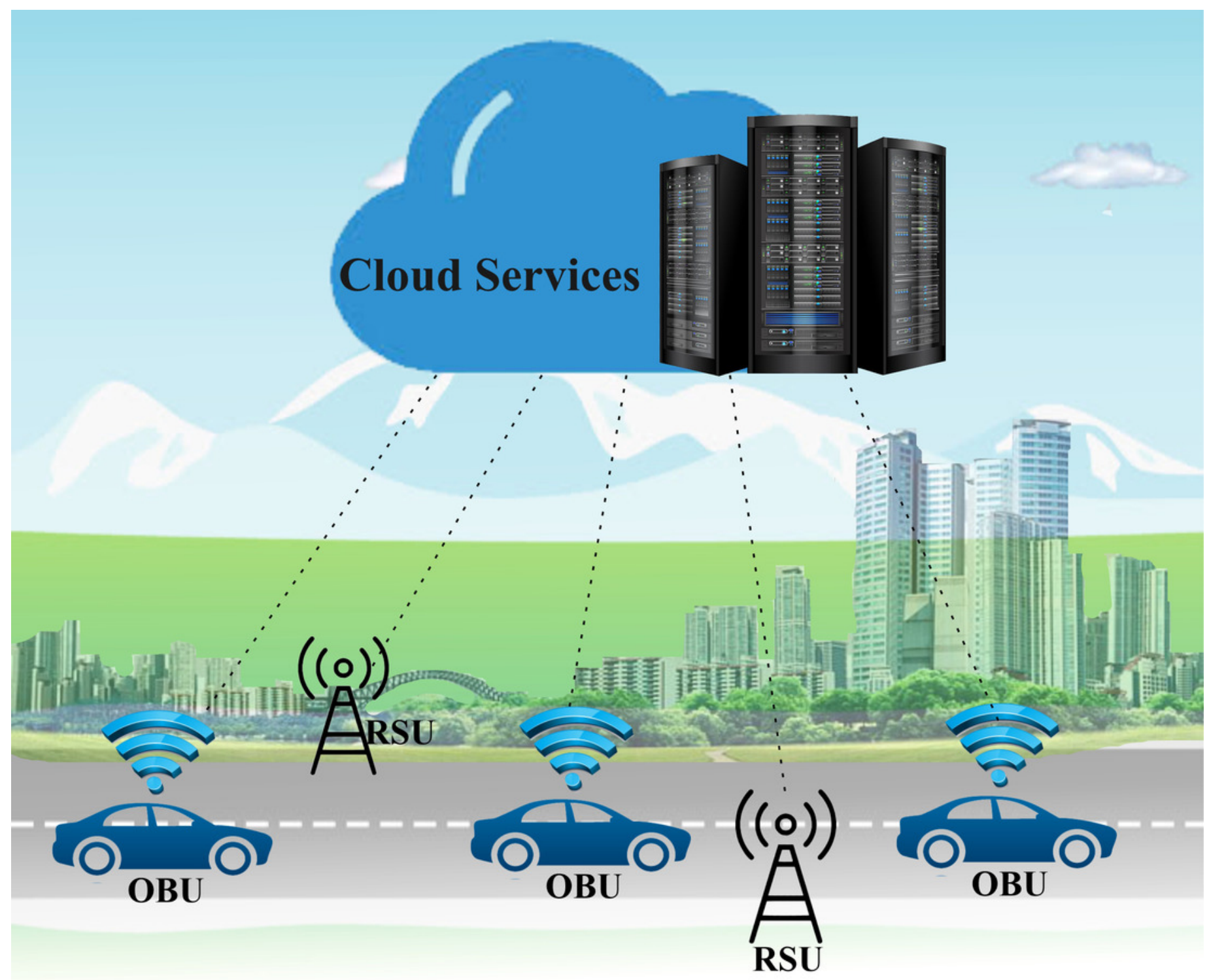


Figure 2

Trust evaluation techniques classified into three types

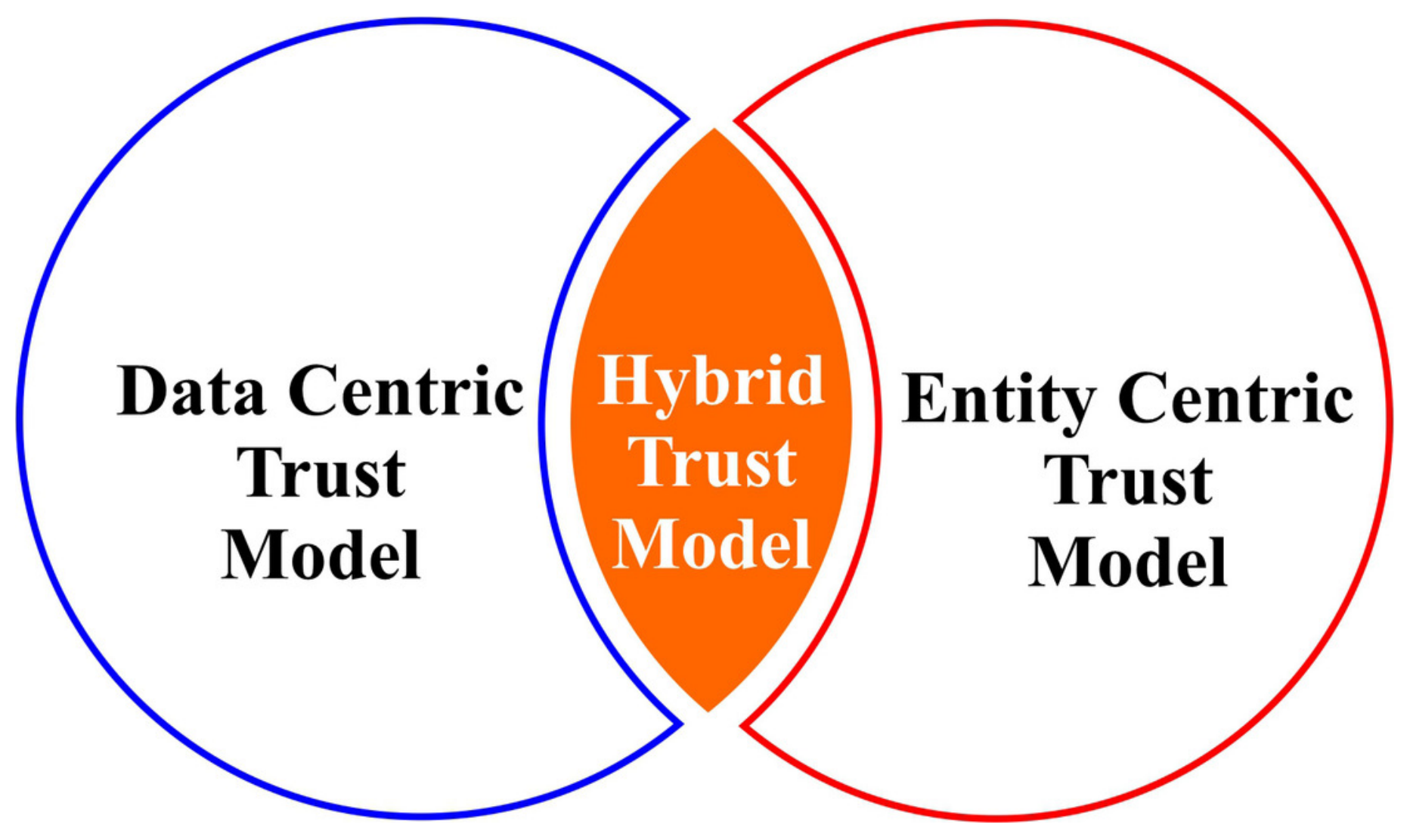


Figure 3

The SLR selection process, and filtering in each phase 


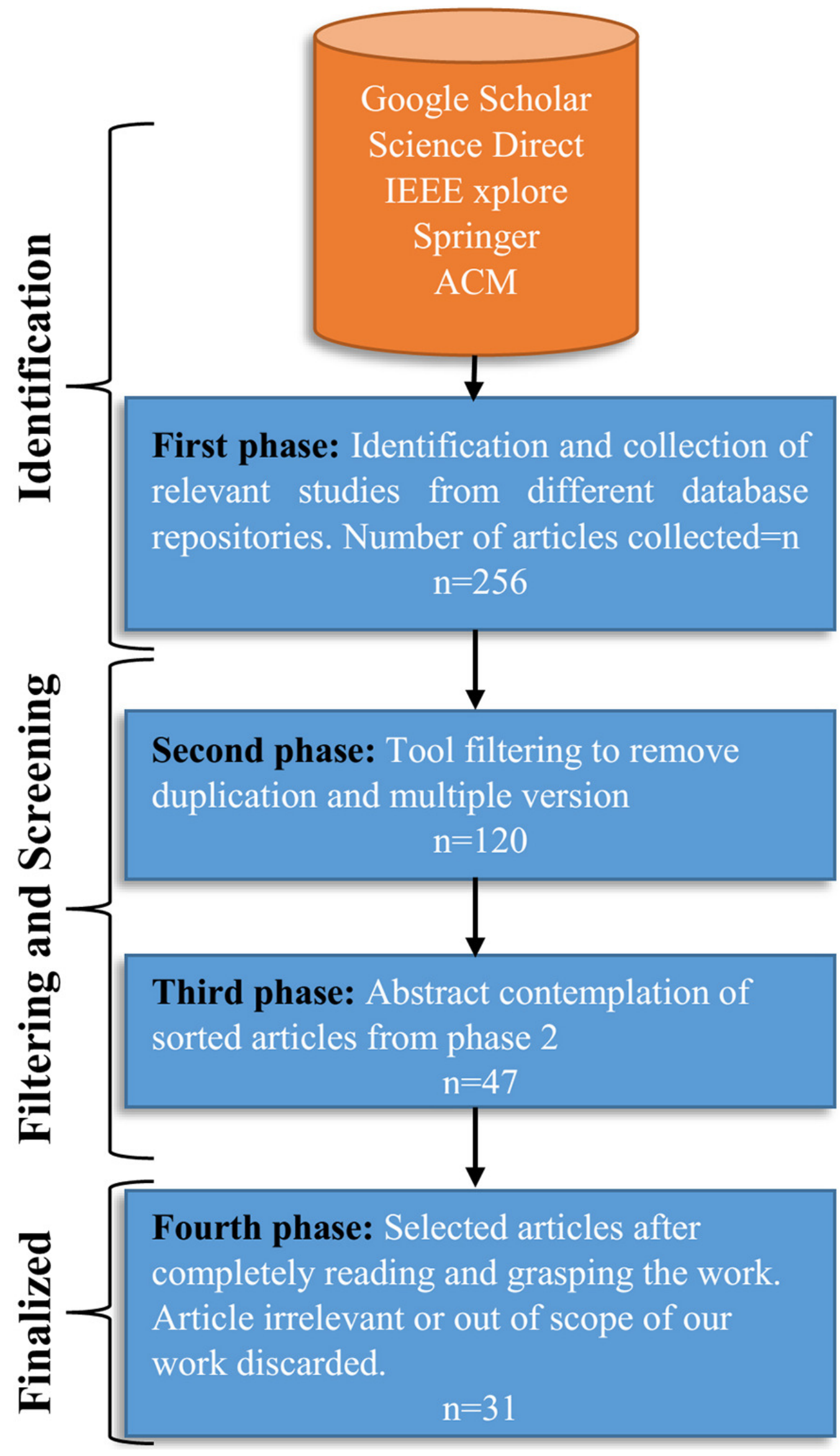


Figure 4

The number of publications on trust management that appeared during a certain decade

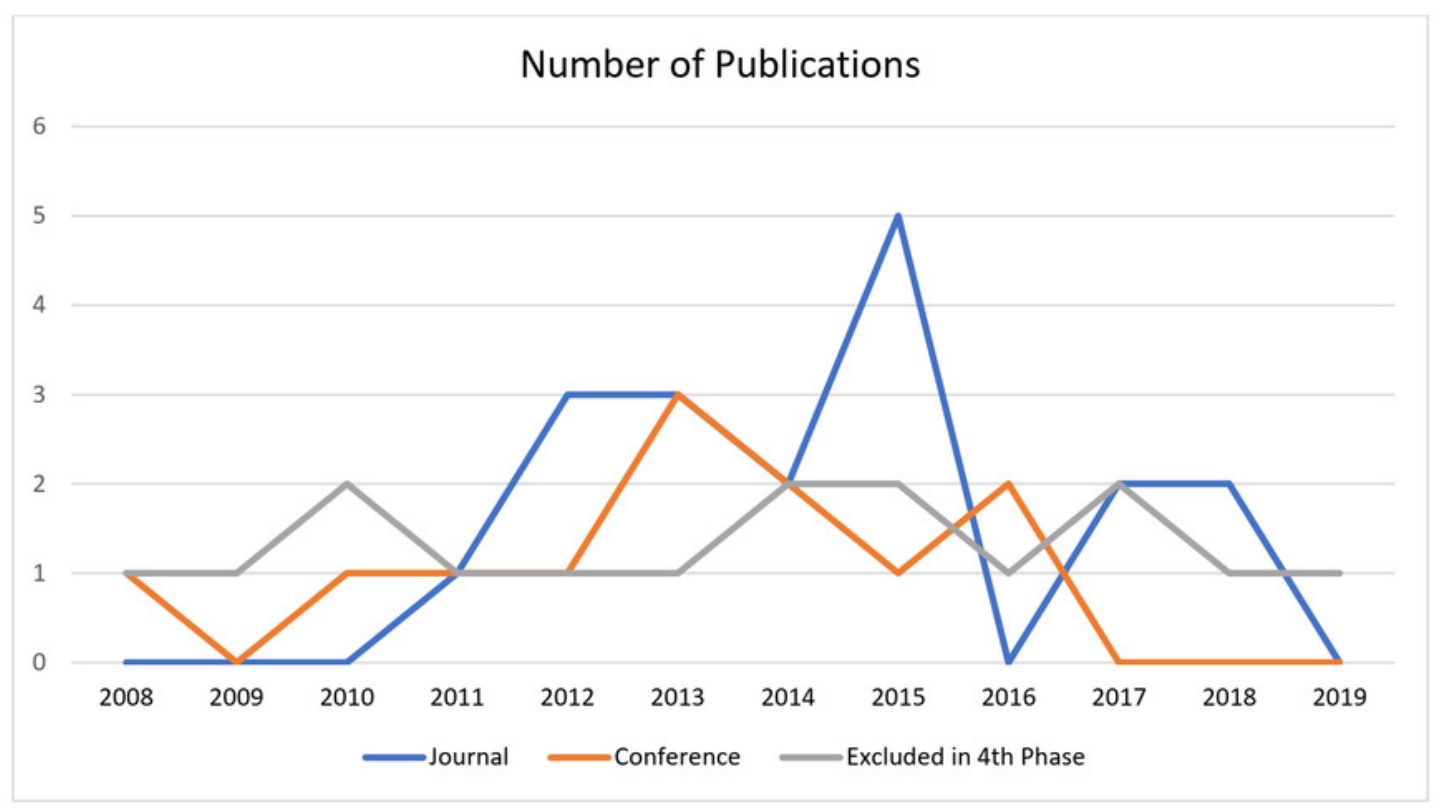


Figure 5

The top ten parameters used in different studies

\section{Commonly Used Perameters}

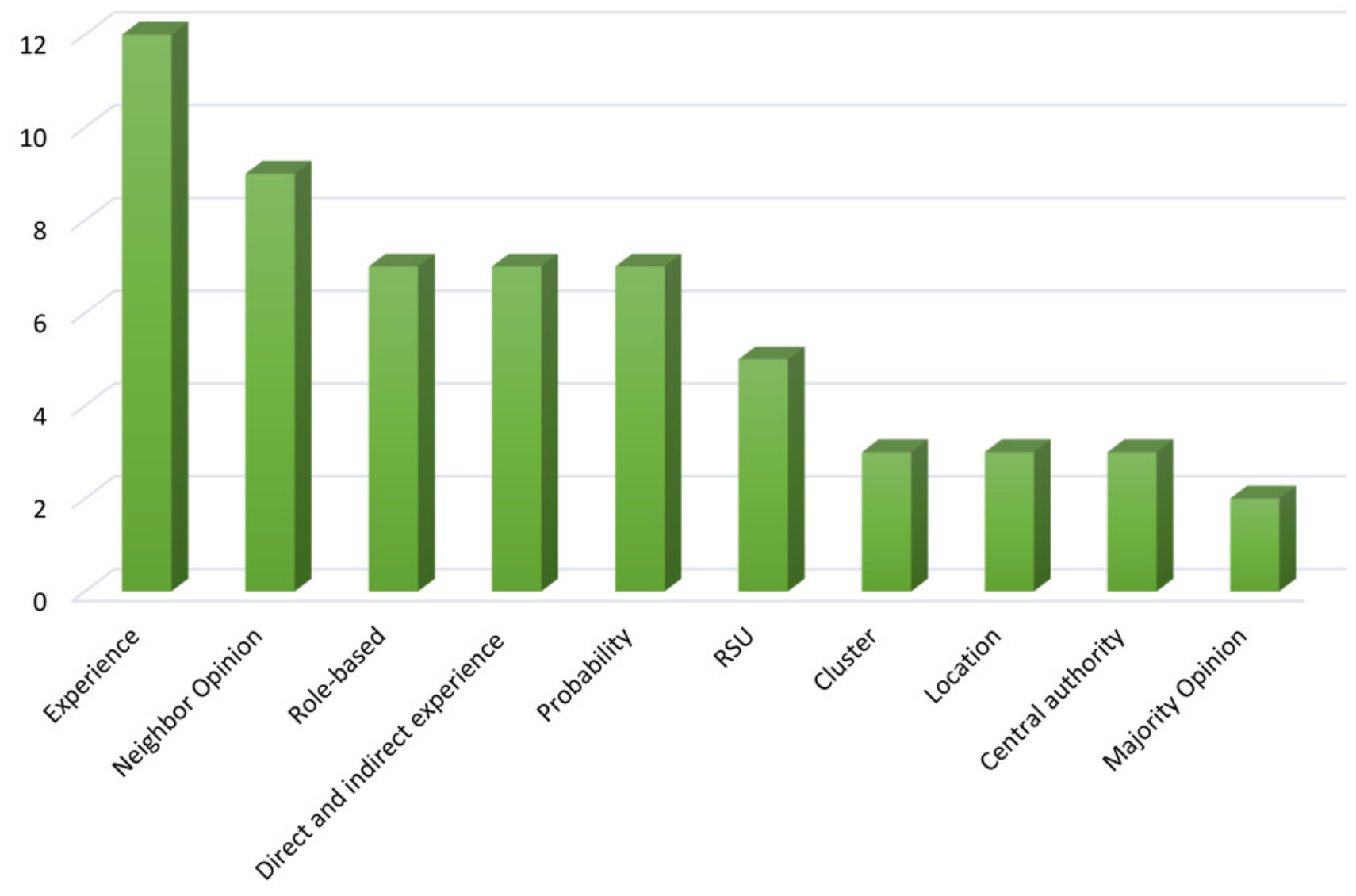




\section{Figure 6}

An overview of the different technologies, the extent to which they overlap, related issues, and the potential future of these technologies

Trust management has become an essential part of vehicular networks

Context awareness: an AI method to incorporate adaption in any rigid system

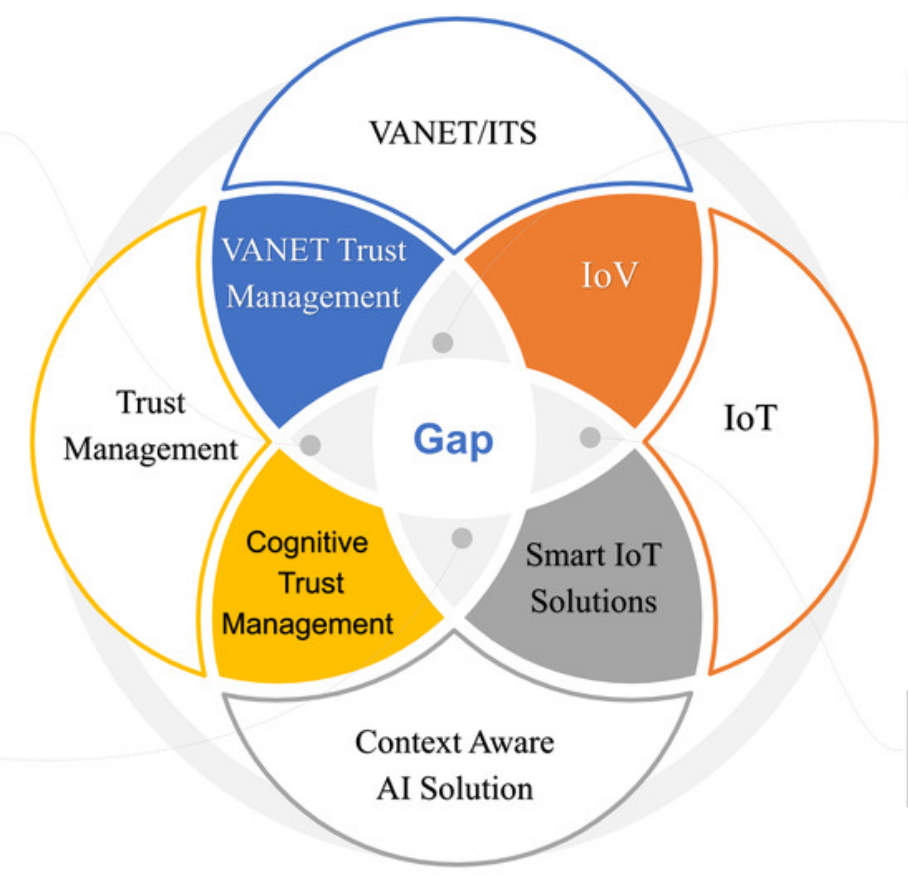

IoT in combination with a vehicular network makes IoV a future component of smart cities 


\section{Table $\mathbf{1}$ (on next page)}

The formulated protocol for this specific study based on the guidelines of a renowned review protocol PRISMA 


\begin{tabular}{|c|c|}
\hline Title & Clear definition of problem with type of study \\
\hline Abstract & $\begin{array}{l}\text { Provide a comprehensive overview of the study being discussed, starting from the } \\
\text { background. Review criteria, methodology, results, and key findings. }\end{array}$ \\
\hline Methodology & $\begin{array}{l}\text { Objectives } \\
\text { Selection criteria } \\
\text { Scope of the study } \\
\text { Limitations }\end{array}$ \\
\hline Introduction & $\begin{array}{l}\text { In the sense of what is already understood, the rationale for the review. A clear statement } \\
\text { of problems being addressed, comparison, outcome and study design }\end{array}$ \\
\hline $\begin{array}{l}\text { Results } \\
\text { Discussion }\end{array}$ & $\begin{array}{l}\text { Discussion on the outcomes with respect to research questions. } \\
\text { Limitations of the study } \\
\text { Open research challenges }\end{array}$ \\
\hline Conclusions & $\begin{array}{l}\text { Summary of the whole study conducted, general understanding of the results, and } \\
\text { suggestions for future research }\end{array}$ \\
\hline
\end{tabular}




\section{Table 2 (on next page)}

The research questions that cover the research objective of the SLR 
1

\begin{tabular}{|l|l|l|}
\hline No. & Research Question & Deliverable/ outcome \\
\hline 1 & $\begin{array}{l}\text { What are the current techniques for trust } \\
\text { evaluation/management in the vehicle } \\
\text { network? }\end{array}$ & $\begin{array}{l}\text { In-depth analysis of current trust evaluation and } \\
\text { management techniques. } \\
\text { Comprehend all the available models in a single study for } \\
\text { further research }\end{array}$ \\
\hline 2 & $\begin{array}{l}\text { To what extent are the proposed models } \\
\text { effective? }\end{array}$ & $\begin{array}{l}\text { Weak and strong properties of current trust models } \\
\text { considering real-time implementation. } \\
\text { Identification of the gray areas of current models, that } \\
\text { need to be investigated } \\
\text { Effectiveness analysis in term of trust evaluation } \\
\text { Open research challenges for researchers. }\end{array}$ \\
\hline 3 & $\begin{array}{l}\text { Is context awareness suitable for the trust } \\
\text { establishment in the vehicle network? }\end{array}$ & $\begin{array}{l}\text { Contrast study of context awareness and vehicular } \\
\text { network. } \\
\text { Enlighten new research dimension for vehicular networks }\end{array}$ \\
\hline
\end{tabular}




\section{Table 3 (on next page)}

the trends of studies conducted for trust evaluation and lists the year 


\begin{tabular}{|c|c|c|c|}
\hline Article & Year & Category & $\begin{array}{r}\text { Journal } \\
\text { /Conference }\end{array}$ \\
\hline (Ahmad et al. 2018) & 2018 & Hybrid & $\mathrm{J}$ \\
\hline (Gazdar et al. 2018) & 2018 & Entity & $\mathrm{J}$ \\
\hline (Soleymani et al. 2017) & 2017 & Entity & $\mathrm{J}$ \\
\hline (Ahmed et al. 2017) & 2017 & Hybrid & $\mathrm{J}$ \\
\hline (Biswas et al. 2016) & 2016 & Hybrid & $\mathrm{C}$ \\
\hline (Hussain et al. 2016) & 2016 & Hybrid & $\mathrm{C}$ \\
\hline (Sedjelmaci \& Senouci 2015) & 2015 & Hybrid & $\mathrm{J}$ \\
\hline (Haddadou et al. 2015) & 2015 & Entity & $\mathrm{J}$ \\
\hline (Rawat et al. 2015) & 2015 & Data & $\mathrm{J}$ \\
\hline (Ya et al. 2015) & 2015 & Entity & $\mathrm{J}$ \\
\hline (Rostamzadeh et al. 2015) & 2015 & Hybrid & $\mathrm{J}$ \\
\hline (Alagar \& Wan 2015) & 2015 & Entity & $\mathrm{C}$ \\
\hline (Abdelaziz et al. 2014) & 2014 & Entity & $\mathrm{C}$ \\
\hline (Wahab et al. 2014) & 2014 & Hybrid & $\mathrm{J}$ \\
\hline (Shaikh \& Alzahrani 2014) & 2014 & Data & $\mathrm{J}$ \\
\hline (Wei et al. 2014) & 2014 & Hybrid & $\mathrm{C}$ \\
\hline (Yang 2013) & 2013 & hybrid & $\mathrm{J}$ \\
\hline (Chen \& Wei 2013) & 2013 & Hybrid & $\mathrm{J}$ \\
\hline (Li et al. 2013) & 2013 & Hybrid & $\mathrm{C}$ \\
\hline (Zhang et al. 2013) & 2013 & Hybrid & $\mathrm{J}$ \\
\hline (Monir et al. 2013) & 2013 & Entity & $\mathrm{C}$ \\
\hline (Rehman et al. 2013) & 2013 & Data & $\mathrm{C}$ \\
\hline (Li et al. 2012) & 2012 & Entity & $\mathrm{J}$ \\
\hline (Gazdar et al. 2012) & 2012 & Hybrid & $\mathrm{C}$ \\
\hline (Sahoo et al. 2012) & 2012 & Entity & $\mathrm{J}$ \\
\hline (Mármol \& Pérez 2012) & 2012 & Entity & $\mathrm{J}$ \\
\hline (Minhas et al. 2011) & 2011 & Entity & $\mathrm{J}$ \\
\hline (Biswas et al. 2011) & 2011 & Entity & $\mathrm{C}$ \\
\hline (Wu et al. 2011) & 2011 & Data & $\mathrm{C}$ \\
\hline (Chen et al. 2010) & 2010 & Data & $\mathrm{C}$ \\
\hline (Raya et al. 2008) & 2008 & Data & $\mathrm{C}$ \\
\hline
\end{tabular}




\section{Table 4 (on next page)}

The important features of the trust model that were identified 


\begin{tabular}{|c|c|c|c|c|}
\hline Article & $\begin{array}{l}\text { CA (Central } \\
\text { Authority) }\end{array}$ & $\begin{array}{l}\text { Authentication/ } \\
\text { PKI }\end{array}$ & $\begin{array}{l}\text { New node } \\
\text { mechanism }\end{array}$ & $\begin{array}{l}\text { Uncertainty } \\
\text { handling }\end{array}$ \\
\hline $\begin{array}{l}\text { (Ahmad et al. } \\
\text { 2018) }\end{array}$ & yes (for role based) & no & 0.5 initial value & no \\
\hline $\begin{array}{l}\text { (Gazdar et al. } \\
\text { 2018) }\end{array}$ & no & no & no & no \\
\hline $\begin{array}{l}\text { (Soleymani et } \\
\text { al. 2017) }\end{array}$ & no & yes & no & no \\
\hline $\begin{array}{l}\text { (Ahmed et al. } \\
\text { 2017) }\end{array}$ & no & yes & no & no \\
\hline $\begin{array}{l}\text { (Biswas et al. } \\
\text { 2016) }\end{array}$ & yes & yes & no & no \\
\hline $\begin{array}{l}\text { (Hussain et } \\
\text { al. 2016) }\end{array}$ & yes & yes & no & no \\
\hline $\begin{array}{l}\text { (Sedjelmaci } \\
\& \text { Senouci } \\
\text { 2015) }\end{array}$ & no & no & no & no \\
\hline $\begin{array}{l}\text { (Haddadou et } \\
\text { al. 2015) }\end{array}$ & no & $\begin{array}{l}\text { RSA, elliptic curve } \\
\text { cryptography }\end{array}$ & no & Markova chain \\
\hline $\begin{array}{l}\text { (Rawat et al. } \\
\text { 2015) }\end{array}$ & no & $\mathrm{x}$ & no & Probabilistic approach \\
\hline $\begin{array}{l}\text { (Ya et al. } \\
\text { 2015) }\end{array}$ & yes & yes & $\begin{array}{l}\text { new node initial } \\
\text { trust value } 0.5\end{array}$ & By location \\
\hline $\begin{array}{l}\text { (Rostamzadeh } \\
\text { et al. 2015) }\end{array}$ & no & no & no & no \\
\hline $\begin{array}{l}\text { (Alagar \& } \\
\text { Wan 2015) }\end{array}$ & yes, CA, GTA & yes & Start with 0 trust & no \\
\hline $\begin{array}{l}\text { (Abdelaziz et } \\
\text { al. 2014) }\end{array}$ & no & no & $\begin{array}{l}\text { new node initial } \\
\text { trust value } 0.5\end{array}$ & no \\
\hline $\begin{array}{l}\text { (Wahab et al. } \\
\text { 2014) }\end{array}$ & no & no & no & Dempster-Shafer \\
\hline $\begin{array}{l}\text { (Shaikh \& } \\
\text { Alzahrani } \\
\text { 2014) }\end{array}$ & no & no & no & no \\
\hline $\begin{array}{l}\text { (Wei et al. } \\
\text { 2014) }\end{array}$ & no & no & no & no \\
\hline (Yang 2013) & no & no & $\begin{array}{l}0.5 \text { (Based on } \\
\text { similar vehicle) }\end{array}$ & no \\
\hline $\begin{array}{l}\text { (Chen \& Wei } \\
\text { 2013) }\end{array}$ & yes & yes & no & $\begin{array}{l}\text { Dempster-Shafer } \\
\text { evidence }\end{array}$ \\
\hline $\begin{array}{l}\text { (Li et al. } \\
\text { 2013) }\end{array}$ & yes, RMC & no & no & no \\
\hline $\begin{array}{l}\text { (Zhang et al. } \\
\text { 2013) }\end{array}$ & yes & no & no & no \\
\hline $\begin{array}{l}\text { (Monir et al. } \\
\text { 2013) }\end{array}$ & yes, GTA & no & Start with 0 & By calculation \\
\hline $\begin{array}{l}\text { (Rehman et } \\
\text { al. 2013) }\end{array}$ & no & no & no & no \\
\hline
\end{tabular}




\begin{tabular}{|c|c|c|c|}
\hline $\begin{array}{l}\text { (Li et al. } \\
2012)\end{array}$ & no & yes & no \\
\hline $\begin{array}{l}\text { (Gazdar et al. } \\
\text { 2012) }\end{array}$ & no & no & no \\
\hline $\begin{array}{l}\text { (Sahoo et al. } \\
\text { 2012) }\end{array}$ & no & no & $\begin{array}{l}\text { All new vehicles are } \\
\text { trusted }\end{array}$ \\
\hline $\begin{array}{l}\text { (Mármol \& } \\
\text { Pérez 2012) }\end{array}$ & yes & no & no \\
\hline $\begin{array}{l}\text { (Minhas et al. } \\
\text { 2011) }\end{array}$ & no & no & no \\
\hline $\begin{array}{l}\text { (Biswas et al. } \\
\text { 2011) }\end{array}$ & no & Local public key & no \\
\hline $\begin{array}{l}\text { (Wu et al. } \\
2011)\end{array}$ & no & no & no \\
\hline $\begin{array}{l}\text { (Chen et al. } \\
\text { 2010) }\end{array}$ & no & no & no \\
\hline (Raya et al. & $\mathrm{CA}$ & PKI & no \\
\hline
\end{tabular}




\section{Table 5 (on next page)}

The simulators used by different trust models 


\begin{tabular}{ll}
\hline Article & Simulator \\
\hline (Ahmad et al. 2018) & VEINS, SUMO, OMNET++ \\
(Gazdar et al. 2018) & not discussed \\
(Soleymani et al. 2017) & NS2, SUMO \\
(Ahmed et al. 2017) & OMNET++ discrete event, network simulator \\
(Biswas et al. 2016) & not discussed \\
(Hussain et al. 2016) & not discussed \\
(Sedjelmaci \& Senouci 2015) & NS-3 \\
(Haddadou et al. 2015) & NS-2, SUMO \\
(Rawat et al. 2015) & not discussed \\
(Ya et al. 2015) & not discussed \\
(Rostamzadeh et al. 2015) & MATLAB \\
(Alagar \& Wan 2015) & not discussed \\
(Abdelaziz et al. 2014) & NS-2 \\
(Wahab et al. 2014) & MATLAB, network simulator, MobiSim traffic \\
(Shaikh \& Alzahrani 2014) & SWANS++ \\
(Wei et al. 2014) & not discussed \\
(Yang 2013) & not discussed \\
(Chen \& Wei 2013) & not discussed \\
(Li et al. 2013) & NA \\
(Zhang et al. 2013) & not discussed \\
(Monir et al. 2013) & MATLAB \\
(Rehman et al. 2013) & not discussed \\
(Li et al. 2012) & Groove Net \\
(Gazdar et al. 2012) & Veins \\
(Sahoo et al. 2012) & not discussed \\
(Mármol \& Pérez 2012) & TRMSim-V2V \\
(Minhas et al. 2011) & SWANS \\
(Biswas et al. 2011) & not discussed \\
(Wu et al. 2011) & NS-3 \\
(Chen et al. 2010) & not discussed \\
(Raya et al. 2008) & MATLAB \\
\hline
\end{tabular}




\section{Table 6 (on next page)}

The review studies in which "vehicular network TM" is discussed 
1

2

\begin{tabular}{lcll}
\hline \multicolumn{1}{c}{ Review study } & Year & \multicolumn{1}{c}{$\begin{array}{c}\text { Type of } \\
\text { publication }\end{array}$} & \multicolumn{1}{c}{ Focus } \\
\hline $\begin{array}{l}\text { (Sumithra \& Vadivel } \\
\text { 2018) }\end{array}$ & 2018 & Conference & Fuzzy logic \\
(Gillani et al. 2018) & 2018 & Conference & Secure routing \\
(Kerrache et al. 2016) & 2016 & Journal & Attacks \\
(Soleymani et al. & 2015 & Journal & Fuzzy logic \\
2015) & & & \\
\hline
\end{tabular}

3 


\section{Table 7 (on next page)}

The core approaches adapted by trust models 


\begin{tabular}{|c|c|}
\hline Article & Approach \\
\hline $\begin{array}{l}\text { (Ahmad et al. 2018; Ahmed et al. 2017; } \\
\text { Chen et al. 2010; Li et al. 2012; Mármol } \\
\text { \& Pérez 2012; Minhas et al. 2011; Monir } \\
\text { et al. 2013; Rostamzadeh et al. 2015; } \\
\text { Sahoo et al. 2012; Soleymani et al. 2017; } \\
\text { Wei et al. 2014; Yang 2013) }\end{array}$ & - Experience-based \\
\hline $\begin{array}{l}\text { (Ahmad et al. 2018; Alagar \& Wan } \\
\text { 2015; Chen et al. 2010; Minhas et al. } \\
\text { 2011; Monir et al. 2013; Raya et al. } \\
\text { 2008; Zhang et al. 2013) }\end{array}$ & - $\quad$ Role-based \\
\hline $\begin{array}{l}\text { (Abdelaziz et al. 2014; Ahmed et al. } \\
\text { 2017; Haddadou et al. 2015; Li et al. } \\
\text { 2013; Mármol \& Pérez 2012; Monir et } \\
\text { al. 2013; Rostamzadeh et al. 2015; } \\
\text { Sahoo et al. 2012; Sedjelmaci \& Senouci } \\
\text { 2015) }\end{array}$ & - Neighbor Opinion \\
\hline $\begin{array}{l}\text { (Ahmed et al. 2017; Biswas et al. 2016; } \\
\text { Chen \& Wei 2013; Gazdar et al. 2018; } \\
\text { Mármol \& Pérez 2012; Wei et al. 2014; } \\
\text { Yang 2013) }\end{array}$ & - Direct and indirect experience \\
\hline $\begin{array}{l}\text { (Chen \& Wei 2013; Gazdar et al. 2012; } \\
\text { Rawat et al. 2015; Raya et al. 2008; } \\
\text { Soleymani et al. 2017; Wahab et al. } \\
\text { 2014; Wei et al. 2014) }\end{array}$ & - Probability, Markova, Dempster-Shafer, Bayesian inference \\
\hline $\begin{array}{l}\text { (Alagar \& Wan 2015; Biswas et al. } \\
\text { 2011; Biswas et al. 2016; Li et al. 2013; } \\
\text { Wu et al. 2011) }\end{array}$ & - $\quad$ RSU managed trust \\
\hline $\begin{array}{l}\text { (Sahoo et al. 2012; Sedjelmaci \& } \\
\text { Senouci 2015; Wahab et al. 2014) }\end{array}$ & - Cluster-based \\
\hline $\begin{array}{l}\text { (Rostamzadeh et al. 2015; Shaikh \& } \\
\text { Alzahrani 2014; Soleymani et al. 2017; } \\
\text { Ya et al. 2015) }\end{array}$ & - Location trust association \\
\hline $\begin{array}{l}\text { (Mármol \& Pérez 2012; Monir et al. } \\
\text { 2013; Zhang et al. 2013) }\end{array}$ & - Trust evaluated by central authority \\
\hline $\begin{array}{l}\text { (Minhas et al. 2011; Sedjelmaci \& } \\
\text { Senouci 2015) }\end{array}$ & - Majority Opinion \\
\hline (Li et al. 2012; Li et al. 2013) & - Centralized trust management \\
\hline $\begin{array}{l}\text { (Gazdar et al. 2018; Haddadou et al. } \\
\text { 2015) }\end{array}$ & - Malicious node detection \\
\hline (Ahmed et al. 2017; Wei et al. 2014) & - Direct trust \\
\hline $\begin{array}{l}\text { (Shaikh \& Alzahrani 2014; Ya et al. } \\
\text { 2015) }\end{array}$ & - Time-based \\
\hline $\begin{array}{l}\text { (Alagar \& Wan 2015; Minhas et al. } \\
\text { 2011) }\end{array}$ & - Agent-based \\
\hline (Soleymani et al. 2017) & $\begin{array}{ll}\text { - } & \text { Fuzzy logic } \\
\text { - } & \text { Local ID authentication }\end{array}$ \\
\hline (Ahmed et al. 2017) & - $\quad$ Attack based model \\
\hline
\end{tabular}


(Ahmad et al. 2018)

(Biswas et al. 2016)

(Hussain et al. 2016)

(Sedjelmaci \& Senouci 2015)

(Haddadou et al. 2015)

(Rawat et al. 2015)

(Ya et al. 2015)

(Abdelaziz et al. 2014)

(Wahab et al. 2014)

(Wei et al. 2014)

(Yang 2013)

(Chen \& Wei 2013)

(Li et al. 2013)

(Zhang et al. 2013)

(Monir et al. 2013)

(Rehman et al. 2013)

(Li et al. 2012)

(Gazdar et al. 2012)

(Sahoo et al. 2012)

(Mármol \& Pérez 2012)

(Minhas et al. 2011)

(Biswas et al. 2011)

(Wu et al. 2011)

(Raya et al. 2008)
- Mobility behavior

- long term trust relationship

- Person(driver) based

- Email-ID associated trust

- Social media

- Attack based

- Credit allocation

- Distributed trust evaluation

- Deterministic

- Response-time

- Continuous link stability

- Piggybacking

- Local trust evaluation

- Hope $(1,2,3)$

- Reward-based

- Recommendation

- Similarity (data, node) theory

- Beacon-based

- Reputation-based

- Peer-based opinion

- Driver ID based trust

- Thread-based

- Reputation score

- Feedback score(opinion)

- Distributed trust management

- Ant colony message routing

- Reward or punishment

- 3 level fuzzy trust

- Decentralized

- ID-based

- Certificate less

- Public key

- Observation reports

- Feedback

- Different trust combined

- Weighted voting 


\section{Table 8(on next page)}

The summary of core techniques, methodologies, and related components used by each TM model 


\begin{tabular}{|c|c|}
\hline Article & Methodology \\
\hline $\begin{array}{l}\text { (Ahmad et al. } \\
\text { 2018) }\end{array}$ & $\begin{array}{l}\text { Trust over data and vehicle is established and finally, message trust is evaluated. } \\
\text { "Role based vehicles" and experience play an important role. }\end{array}$ \\
\hline $\begin{array}{l}\text { (Gazdar et al. } \\
\text { 2018) }\end{array}$ & $\begin{array}{l}\text { Neighboring-node opinion is considered. Negative trust introduced in this model. Probability is } \\
\text { used for uncertainty handling. }\end{array}$ \\
\hline $\begin{array}{l}\text { (Soleymani et } \\
\text { al. 2017) }\end{array}$ & $\begin{array}{l}\text { Trust determined by fuzzy logic, experience, and probability. Local ID authentication. } \\
\text { Experience value is saved in the node. Location verification using time and distance. The sender } \\
\text { location is a key factor in this model. }\end{array}$ \\
\hline $\begin{array}{l}\text { (Ahmed et al. } \\
\text { 2017) }\end{array}$ & $\begin{array}{l}\text { Neighbor opinion, consistency, and similarity-based trust evaluation. Three subcategories of } \\
\text { trust: events, nodes, and recommendations. }\end{array}$ \\
\hline $\begin{array}{l}\text { (Biswas et al. } \\
\text { 2016) }\end{array}$ & $\begin{array}{l}\text { RSU add and manage trust. Long term trust relationship is maintained. RSU supported by CA at } \\
\text { the top layer. The model assumes that all the vehicles in the networks registered with the CA and } \\
\text { maintains a certain level of trust. }\end{array}$ \\
\hline $\begin{array}{l}\text { (Hussain et } \\
\text { al. 2016) }\end{array}$ & Based on email and social trust. Trust is managed by a CA. Trust is associated with user. \\
\hline $\begin{array}{l}\text { Sedjelmaci } \\
\& \text { Senouci } \\
2015)\end{array}$ & $\begin{array}{l}\text { Three categories of trust (trusted, suspicious and attacker). Majority voting-based trust. The } \\
\text { cluster head is responsible for trust management. }\end{array}$ \\
\hline $\begin{array}{l}\text { (Haddadou et } \\
\text { al. 2015) }\end{array}$ & $\begin{array}{l}\text { Credit-based model removes malicious nodes. Selfish nodes get participation rewards. RSA } \\
\text { cryptographic scheme used. }\end{array}$ \\
\hline $\begin{array}{l}\text { (Rawat et al. } \\
\text { 2015) }\end{array}$ & $\begin{array}{l}\text { The trust evaluated by calculating distances using signal-strength, arrival-time and location. The } \\
\text { probability of a malicious node is calculated by Bayesian. }\end{array}$ \\
\hline $\begin{array}{l}\text { (Ya et al. } \\
2015)\end{array}$ & $\begin{array}{l}\text { A time frame-based trust protocol. Feedback on report from neighboring nodes. Selfish and } \\
\text { malicious node identification. To validate the trust response time is used. }\end{array}$ \\
\hline $\begin{array}{l}\text { (Rostamzade } \\
\text { h et al. 2015) }\end{array}$ & $\begin{array}{l}\text { The trust model is subdivided in multiple modules. Three security levels to assure trust. Trust is } \\
\text { allocated to road segments. }\end{array}$ \\
\hline $\begin{array}{l}\text { (Alagar \& } \\
\text { Wan 2015) }\end{array}$ & $\begin{array}{l}\text { Role-based vehicles act as agents. RSU assesses the context environment and manages the } \\
\text { system. }\end{array}$ \\
\hline $\begin{array}{l}\text { (Abdelaziz et } \\
\text { al. 2014) }\end{array}$ & $\begin{array}{l}\text { The trust model is subdivided in multiple modules. Three security levels to assure trust. Trust is } \\
\text { allocated to road segments. }\end{array}$ \\
\hline $\begin{array}{l}\text { (Wahab et al. } \\
\text { 2014) }\end{array}$ & $\begin{array}{l}\text { Role-based vehicles act as agents. RSU assesses the context environment and manages the } \\
\text { system. }\end{array}$ \\
\hline $\begin{array}{l}\text { (Shaikh \& } \\
\text { Alzahrani } \\
\text { 2014) }\end{array}$ & $\begin{array}{l}\text { Neighbor vehicles chose the most reliable nodes among adjacent nodes to be used for hopping. } \\
\text { Local trust management by each vehicle. Piggybacking is used by the last message. }\end{array}$ \\
\hline $\begin{array}{l}\text { (Wei et al. } \\
\text { 2014) }\end{array}$ & $\begin{array}{l}\text { Cluster-based trust model. Misbehaving vehicles detected by speed variation. Reward allowed } \\
\text { for motivation. Dempster-Shafer theory for counter verification. Some nodes act as watchdog in } \\
\text { the system. }\end{array}$ \\
\hline (Yang 2013) & $\begin{array}{l}\text { A decentralized trust management system. The scheme is based on change in the location and } \\
\text { time used to calculate the trust. }\end{array}$ \\
\hline $\begin{array}{l}\text { (Chen \& Wei } \\
\text { 2013) }\end{array}$ & $\begin{array}{l}\text { The Bayesian rule method is used to calculate the trust level. Dempster-Shafer is used for } \\
\text { handling uncertainty }\end{array}$ \\
\hline $\begin{array}{l}\text { (Li et al. } \\
2013)\end{array}$ & $\begin{array}{l}\text { Nodes monitor neighboring nodes. Trust is based on opinion. Final trust is calculated from new } \\
\text { and previous data. RSU is the part of trust evaluation. }\end{array}$ \\
\hline $\begin{array}{l}\text { (Zhang et al. } \\
\text { 2013) }\end{array}$ & $\begin{array}{l}\text { Before forwarding received messages, peer inputs their opinion. Role-based nodes are also part } \\
\text { of the model. The central authority manages the whole network }\end{array}$ \\
\hline
\end{tabular}




\begin{tabular}{|c|c|}
\hline $\begin{array}{l}\text { (Monir et al. } \\
\text { 2013) }\end{array}$ & $\begin{array}{l}\text { Association of driver ID with trust. CA and RSU are responsible for opinion gathering and trust } \\
\text { evaluation. A malicious node penalizing system introduced. }\end{array}$ \\
\hline $\begin{array}{l}\text { (Rehman et } \\
\text { al. 2013) }\end{array}$ & $\begin{array}{l}\text { During the hopping process trust level is increased. The multiple chains of trust threads created } \\
\text { to finally evaluate trust. }\end{array}$ \\
\hline $\begin{array}{l}\text { (Li et al. } \\
\text { 2012) }\end{array}$ & $\begin{array}{l}\text { The trust is observed as a reputation score of any node network. Experience is involved in } \\
\text { building a reputation. Feedback is recorded against the reporting vehicle. The centralized } \\
\text { authority shall serve as controlling authority }\end{array}$ \\
\hline $\begin{array}{l}\text { (Gazdar et al. } \\
\text { 2012) }\end{array}$ & $\begin{array}{l}\text { Based on vehicle behavior using a Markov chain. All vehicles monitor their surroundings. Local } \\
\text { trust management. }\end{array}$ \\
\hline $\begin{array}{l}\text { (Sahoo et al. } \\
\text { 2012) }\end{array}$ & $\begin{array}{l}\text { An ant colony-based cluster trust model. The cluster is made using different parameters such as } \\
\text { direction, speed, and others. Higher trust level and neighbor's opinion decide cluster head. }\end{array}$ \\
\hline $\begin{array}{l}\text { (Mármol \& } \\
\text { Pérez 2012) }\end{array}$ & $\begin{array}{l}\text { Prior experience, neighbor suggestion are the main trust evaluators in this model with three } \\
\text { levels of local trust storage. CA managed node trust. The reward and punishment system. The } \\
\text { trust is determined in a fuzzy system. }\end{array}$ \\
\hline $\begin{array}{l}\text { (Minhas et al. } \\
\text { 2011) }\end{array}$ & $\begin{array}{l}\text { An intelligent agent-based approach. Trust based on experience, role, priority and majority } \\
\text { opinion. }\end{array}$ \\
\hline $\begin{array}{l}\text { (Biswas et al. } \\
\text { 2011) }\end{array}$ & $\begin{array}{l}\text { The model is based on node ID. Nodes are identified by the digital signatures. Public key } \\
\text { verification is used for authentication. }\end{array}$ \\
\hline $\begin{array}{l}\text { (Wu et al. } \\
2011)\end{array}$ & $\begin{array}{l}\text { An RSU based trust model. The RSU is responsible for trust evaluation. Feedback process adds } \\
\text { up to trustworthiness. }\end{array}$ \\
\hline $\begin{array}{l}\text { (Chen et al. } \\
2010)\end{array}$ & A role-based trust model. Experience and neighbor node opinion. \\
\hline $\begin{array}{l}\text { (Raya et al. } \\
2008 \text { ) }\end{array}$ & $\begin{array}{l}\text { The distributed trust model is based on role-based desperation. Government-related vehicles hold } \\
\text { a higher trust level. Bayesian inference and Dempster-Shafer theory are used to handle } \\
\text { uncertainty. Event-specific trust is observed. }\end{array}$ \\
\hline
\end{tabular}




\section{Table 9 (on next page)}

The assumptions made by the trust models 


\section{Article Assumptions made by the studies}

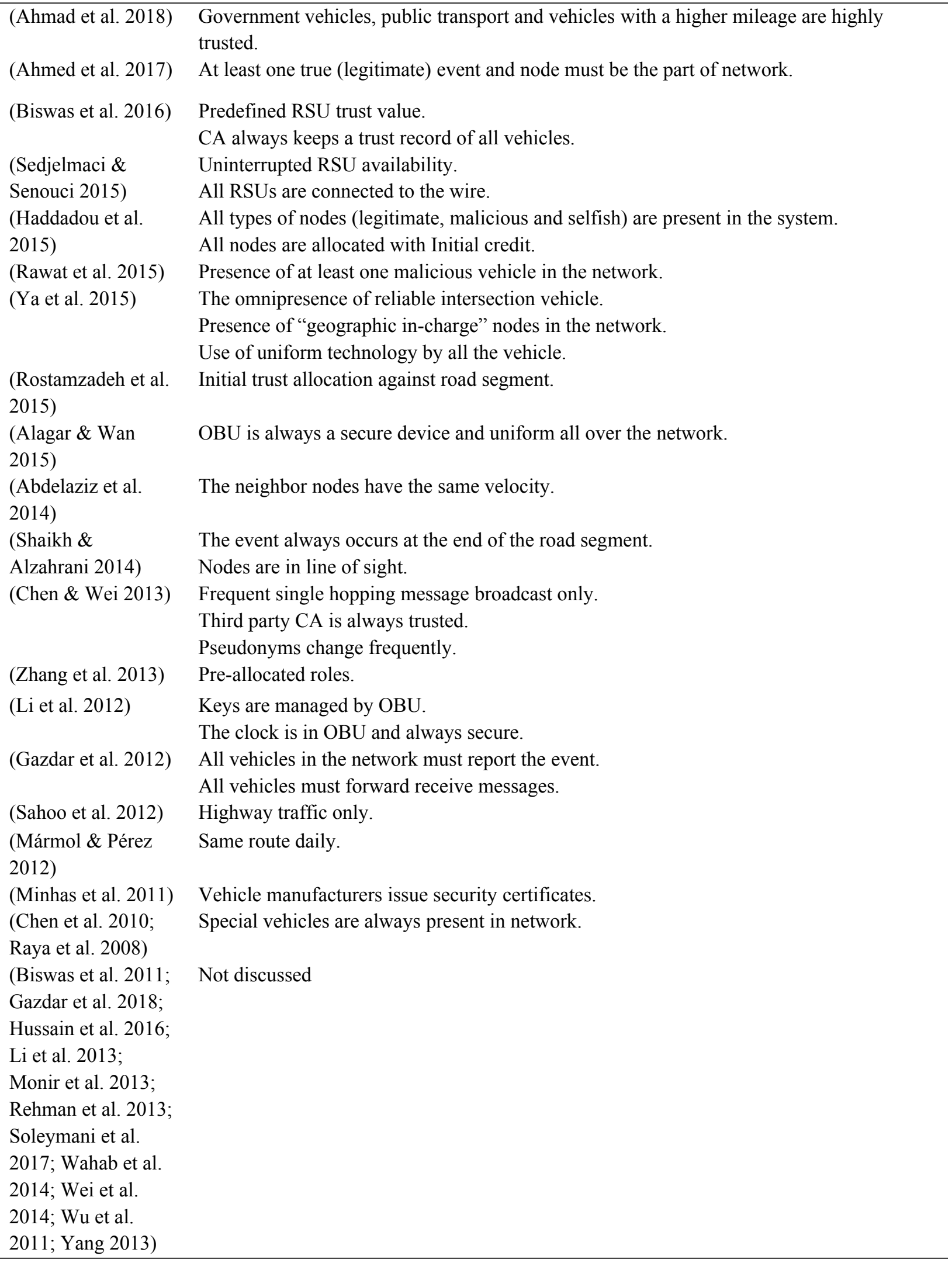




\section{Table $\mathbf{1 0}$ (on next page)}

A comparative overview of VANET and MANET 
1

\begin{tabular}{lll}
\hline \multicolumn{1}{c}{ Property } & \multicolumn{1}{c}{ VANET } & \multicolumn{1}{c}{ MANET } \\
\hline Mobility & High & Low \\
Size & Large & Small \\
Movement & Geographic paths & Random in limited space \\
Protocol & $802.11 \mathrm{p}$ & $802.11 \mathrm{a}$ \\
Topology & Fast-changing & Slow changing \\
Power & Unlimited & Limited \\
Node speed & Very high & low \\
Interaction time & low & High \\
No of nodes & Unknown & Can be restricted \\
Anonymity & High & Low \\
Node variation & Very High & Low \\
\hline
\end{tabular}

2

3 


\section{Table $\mathbf{1 1}$ (on next page)}

summarizes the major properties of the trust models (only journal articles were considered) 


\begin{tabular}{|c|c|c|c|c|c|c|c|c|c|c|c|c|c|c|c|}
\hline$\frac{7}{\stackrel{7}{己}}$ & $\overleftarrow{\mho}$ & 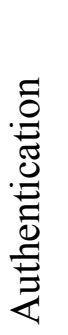 & 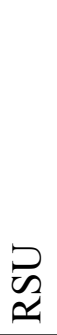 & 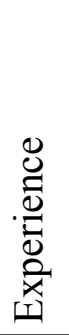 & 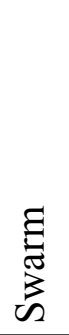 & 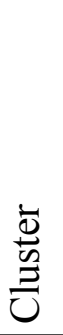 & .0ْ & 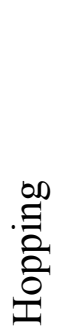 & 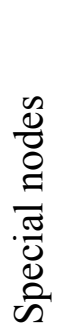 & 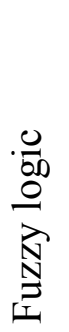 & 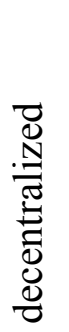 & 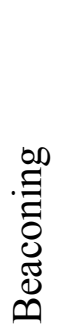 & 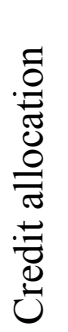 & 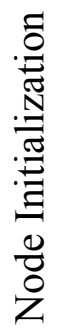 & 忌 \\
\hline $\begin{array}{l}\text { (Ahmad } \\
\text { et al. } \\
2018 \text { ) }\end{array}$ & yes & no & no & yes & no & no & no & no & yes & no & no & no & no & yes & no \\
\hline $\begin{array}{l}\text { (Gazdar } \\
\text { et al. } \\
2018 \text { ) }\end{array}$ & no & no & no & yes & no & no & no & no & no & no & no & no & no & no & no \\
\hline $\begin{array}{l}\text { (Soleyma } \\
\text { ni et al. } \\
2017 \text { ) }\end{array}$ & no & yes & no & yes & no & no & no & no & no & yes & no & no & no & no & no \\
\hline $\begin{array}{l}\text { (Ahmed } \\
\text { et al. } \\
2017 \text { ) }\end{array}$ & no & yes & no & yes & no & no & yes & no & no & no & no & no & no & no & no \\
\hline $\begin{array}{l}\text { (Wahab } \\
\text { et al. } \\
2014 \text { ) }\end{array}$ & no & no & no & no & no & no & no & no & no & no & no & no & yes & no & yes \\
\hline $\begin{array}{l}\text { (Shaikh } \\
\& \\
\text { Alzahran } \\
\text { i 2014) }\end{array}$ & no & no & no & no & no & no & no & no & no & no & yes & no & no & no & no \\
\hline $\begin{array}{l}\text { (Yang } \\
2013 \text { ) }\end{array}$ & no & no & no & yes & no & no & no & no & no & no & no & no & no & yes & no \\
\hline $\begin{array}{l}\text { (Chen \& } \\
\text { Wei } \\
2013)\end{array}$ & yes & yes & no & no & no & no & yes & yes & no & no & no & no & no & no & yes \\
\hline $\begin{array}{l}\text { (Zhang et } \\
\text { al. 2013) }\end{array}$ & yes & no & no & no & no & no & yes & no & yes & no & no & no & no & no & no \\
\hline $\begin{array}{l}\text { (Li et al. } \\
2012)\end{array}$ & no & yes & no & yes & no & no & yes & no & no & no & no & no & no & no & no \\
\hline $\begin{array}{l}\text { (Sahoo et } \\
\text { al. 2012) }\end{array}$ & no & no & no & yes & yes & yes & yes & no & no & no & no & no & no & yes & no \\
\hline $\begin{array}{l}\text { (Mármol } \\
\text { \& Pérez } \\
\text { 2012) }\end{array}$ & yes & no & no & yes & no & no & yes & no & no & yes & no & no & yes & no & no \\
\hline $\begin{array}{l}\text { (Minhas } \\
\text { et al. } \\
2011 \text { ) }\end{array}$ & no & no & no & yes & no & no & yes & no & no & no & yes & no & no & no & no \\
\hline
\end{tabular}

1 


\section{Table $\mathbf{1 2}$ (on next page)}

research challenges and directions related to gray areas 


\begin{tabular}{|c|c|c|c|}
\hline $\begin{array}{l}\text { S. } \\
\text { No. }\end{array}$ & $\begin{array}{l}\text { Related } \\
\text { domain }\end{array}$ & Challenge & Research direction \\
\hline 1. & $\begin{array}{l}\mathrm{TM} \\
\text { frameworks }\end{array}$ & Development of Intelligent TM & $\begin{array}{l}\text { Trust management requires intelligent solutions, } \\
\text { intelligent solutions in trust management are missing. }\end{array}$ \\
\hline 2. & Network & $\begin{array}{l}\text { Choosing between a centralized or } \\
\text { decentralized network }\end{array}$ & $\begin{array}{l}\text { A comparative study is required between centralized } \\
\text { and decentralized approaches for ad hoc networks. }\end{array}$ \\
\hline 3. & $\begin{array}{l}\text { Intelligent } \\
\text { computing }\end{array}$ & $\begin{array}{l}\text { Implementing concepts of nature- } \\
\text { inspired computing (NIC) in } \\
\text { vehicular ad hoc networks }\end{array}$ & $\begin{array}{l}\text { The vehicular network has similarities with nature- } \\
\text { inspired computing such as swarm computing and ant } \\
\text { colony. The scientific coherence needs to be } \\
\text { explored. }\end{array}$ \\
\hline 4. & $\begin{array}{l}\text { Network } \\
\text { initialization }\end{array}$ & $\begin{array}{l}\text { Introducing a new node in ad hoc } \\
\text { network }\end{array}$ & $\begin{array}{l}\text { While managing the trust new node entry in a } \\
\text { network is a problem that needs to be addressed. }\end{array}$ \\
\hline 5. & $\begin{array}{l}\text { Network } \\
\text { cluster }\end{array}$ & Cluster management & $\begin{array}{l}\text { The cluster formatting and management for vehicular } \\
\text { network needs to be standardized. }\end{array}$ \\
\hline 6. & Uncertainty & $\begin{array}{l}\text { Handling uncertainty while } \\
\text { managing trust }\end{array}$ & $\begin{array}{l}\text { One of the complex problems while working on trust } \\
\text { evaluation is uncertainty. Comprehensive solutions } \\
\text { are required to handle uncertainty }\end{array}$ \\
\hline 7. & Social media & Use of social media for security & $\begin{array}{l}\text { The role of social media in enhancing the vehicular } \\
\text { network security needs to be explored }\end{array}$ \\
\hline 8. & Cybersecurity & Preventing cyber attacks & $\begin{array}{l}\text { The greatest threat to IoV security is cyber-attacks. } \\
\text { IoV desperately requires cybersecurity protocols. }\end{array}$ \\
\hline 9. & $\begin{array}{l}\text { Artificial } \\
\text { intelligence }\end{array}$ & $\begin{array}{l}\text { Use of context-awareness in ad } \\
\text { hoc networks }\end{array}$ & $\begin{array}{l}\text { The use of context awareness in ad hoc network } \\
\text { security is a huge research area, that needs to be } \\
\text { explored }\end{array}$ \\
\hline 10. & $\begin{array}{l}\text { Context } \\
\text { awareness }\end{array}$ & $\begin{array}{l}\text { Implementation and knowledge } \\
\text { representing }\end{array}$ & $\begin{array}{l}\text { Implementing context awareness is a huge research } \\
\text { area, especially knowledge representation in ad hoc } \\
\text { networks. An intense in-depth research exercise is } \\
\text { required in this direction. }\end{array}$ \\
\hline
\end{tabular}

